\title{
Epigenetic identification of ADCY4 as a biomarker for breast cancer: an integrated analysis of adenylate cyclases
}

\author{
Yu Fan*,1, Junhao Mu², Mingquan Huang ${ }^{3}$, Saber Imani ${ }^{1}$, Yu Wang ${ }^{4}$, Sheng Lin ${ }^{1}$, Juan \\ Fan $^{1}$ \& Qinglian Wen ${ }^{1}$ \\ ${ }^{1}$ Oncology Department, The Affiliated Hospital of Southwest Medical University, 646000 Luzhou, PR China \\ ${ }^{2}$ Chongqing Key Laboratory of Molecular Oncology \& Epigenetics, The First Affiliated Hospital of Chongqing Medical University, \\ 400010 Chongqing, PR China \\ ${ }^{3}$ Breast Surgery Department, The Affiliated Hospital of Southwest Medical University, 646000 Luzhou, PR China \\ ${ }^{4}$ Health Examination Department, The Affiliated Hospital of Southwest Medical University, 646000 Luzhou, PR China \\ *Author for correspondence: Tel.. +86 15283035180; Fax: +86 830-3165396; yufan@swmu.edu.cn
}

Aim: To explore the role of adenylyl cyclase isoforms and its epigenetics in cancer. Materials \& methods: Adenylyl cyclase expression profiles, epigenetic alterations, prognostic value and molecular networks were assessed by use of public omics datasets. Results: $A D C Y 4$ was significantly downregulated in breast cancer. This downregulation was associated with promoter hypermethylation. High ADCY4 expression was correlated with better survival of patients with breast cancer and its different intrinsic subtypes and tumor stages. ADCY4 was shown to be strongly associated with $G$ protein coupled receptors and the downstream CAMP signaling pathway, which was also significantly enriched in newly identified lysophosphatidic acid receptor 4 and glucagon-like peptide-1. Conclusion: ADCY4 may be used as an epigenetic biomarker for breast cancer, as well as a possible target for therapy.

First draft submitted: 19 July 2019; Accepted for publication: 5 September 2019; Published online: 4 October 2019

Keywords: adenylyl cyclases $\bullet$ biomarker $\bullet$ breast cancer $\bullet$ CAMP signaling pathway $\bullet$ DNA methylation $\bullet$ G proteincoupled receptor $\bullet$ lysophosphatidic acid receptor $\bullet$ prognosis

Cancer has become as a serious threat to human health worldwide. Lung cancer is the most commonly diagnosed cancer and the leading cause of cancer death. It is closely followed by breast cancer for incidence and colorectal cancer, stomach cancer and liver cancer for mortality. These estimates of cancer incidence and mortality are based on GLOBOCAN 2018, a status report on the global burden of cancer [1]. Human cancer is a highly heterogeneous and complex disease [2], for which new treatment options are urgently needed. Notably, treatment targeting of cAMP signaling has been shown to have a number of antitumor effects, including the induction of mesenchymalto-epithelial transition, inhibition of cell growth and migration, as well as improvements in conventional antitumor drug sensitivity [3-5].

The cAMP signaling pathway is defined by spatial and temporal organization of adenylyl cyclases (ACs). cAMP derived from specific ACs can selectively and differentially regulate effector proteins due to distinct tissue distribution of specific AC isoforms. These isoforms often integrate opposing cellular signals and serve as key enzymes for signaling pathways [6]. ADCY7 deficiency results in growth inhibition, increased apoptosis and decreased c-Myc expression in leukemic cells [7]. One study has shown $A D C Y 3$ to be regulated by DNA methylation. ADCY3 is an oncogene for gastric cancer and may be the molecular basis for ADCY3 carcinogenesis. These results provide a rationale for the study of new molecular gastric cancer targets and suggest that DNA methylation plays an important role in the expression and regulation of some AC isoforms [8]. However, there is a lack of evidence for a specific biologic function for ACs in malignant tumors, with a clear need to identify roles for AC isoforms in tumor development and progression. Furthermore, DNA methylation plays a novel role in epigenetic regulation of gene transcription, with high levels of promoter methylation known to stabilize epigenetic gene repression [9]. As such, a consideration of epigenetics with regard to the role of AC isoforms in human cancer is essential.

Future Medicine 
In this study, $A C$ mRNA expression and related DNA methylation in human cancers were assessed based on public datasets. We also evaluated the prognostic value and potential regulatory networks of specific ACs involved in tumorigenesis and tumor progression.

\section{Materials \& methods}

The mRNA expression investigation

The mRNA levels of $A C s$ in human cancers were analyzed using Oncomine microarray datasets (http://www.oncomi ne.org) [10]. The cut-off of p-value, fold change and gene rank were $0.0001,2$ and $10 \%$ in this analysis, respectively. In order to investigate the gene expression in various subgroups with different clinicopathologic parameters, a web portal UALCAN (http://ualcan.path.uab.edu/) based on TCGA datasets was used to analyze the mRNA expressions in human cancers and in their different grades and stages [11].

\section{The analysis of DNA methylation modifications}

To further plot the regulatory effect of DNA methylation modification in gene mRNA expression, the MethHC (http://methhc.mbc.nctu.edu.tw/) that contained 839 breast cancer samples and others was used to investigate the association with promoter methylation and mRNA expression for specific $A C$ isoform [12]. Then, 871 samples of breast invasive carcinoma originated from MEXPRESS (https://mexpress.be/) datasets were analyzed to evaluate the $A D C Y 4$ gene [13].

\section{Genetic alterations evaluation}

An open access genomics resource cBioPortal (www.cbioportal.org) is used to explore the interactive and multidimensional cancer datasets [14]. The genetic alterations of $A D C Y 4$ were evaluated from invasive breast carcinoma (TCGA, Provisional, 1108 samples). The copy number alterations of ADCY4 in human cancers were further identified by a web portal (https://portals.broadinstitute.org/).

\section{Kaplan-Meier survival analysis}

The association with the specific $A C$ expression and clinical prognosis was evaluated based on Kaplan-Meier (KM) plotter (http://kmplot.com/) [15]. We selected the survival of 'RFS' (relapse-free survival) and 'OS' (overall survival), 'Auto-select best cut-off' and 'Split patients by median' to plot the different survival analysis curves. The log-rank method was used to determine the statistic differences and hazard ratios (HRs) with 95\% CIs. The Affymetrix probeset ID of $A D C Y 3$ is 209320_at in breast cancer, and 230800_at for ADCY4 in lung cancer. The RNA-Seq ID of $A D C Y 1$ is 107 in liver cancer.

\section{Initially search for gene regulatory networks}

Functional regulatory networks for ADCY4 was determined by the STRING (Search Tool for the Retrieval of Interacting Genes/Proteins; https://string-db.org/) [16]. The confidence score is defined as 0.4. Metascape (http://metascape.org/) was applied to the analysis of gene annotation and pathway enrichment of ADCY4associated genes [17].

\section{Results}

mRNA expression of $A C$ isoforms in human cancers

To identify roles for ACs in tumorigenesis, we first assessed Oncomine datasets for $A C$ isoform RNA expression in a wide variety of human cancer types. mRNA expression levels of several $A C$ isoforms were aberrantly expressed in human cancers, especially in breast cancer and colorectal cancer (Figure 1). Compared with normal tissues, the expression of $A D C Y 4$ mRNA was significantly downregulated in breast cancer (12 datasets [18-20]). ADCY9 was decreased in colorectal cancer (11 datasets [21-24]) and lung cancer (nine datasets [25-29]) when compared with normal tissues. In contrast, $A D C Y 4$ was markedly increased in the stroma of invasive breast carcinoma with a 2.27-fold change in Finak's dataset (Table 1) [18].

$A D C Y 4$ and $A D C Y 9 \mathrm{mRNA}$ expression was confirmed in invasive breast carcinoma, colon adenocarcinoma, rectal adenocarcinoma, lung adenocarcinoma and lung squamous cell carcinoma using UALCAN. We found ADCY4 and $A D C Y 9$ to be significantly abnormal in these human cancers. Among them, $A D C Y 4$ was downregulated 4.12fold $(\mathrm{p}<1.00 \mathrm{e}-12)$ (Figure $2 \mathrm{~A}$ ) in primary breast cancer, compared with normal tissue. Similarly, $A D C Y 9$ was downregulated 2.55-fold and 2.58-fold in colon and rectal cancer, respectively $(\mathrm{p}=1.63 \mathrm{E}-12$ and $\mathrm{p}=4.90 \mathrm{E}-03$, 


\begin{tabular}{|c|c|c|c|c|c|c|c|c|c|c|c|c|c|c|c|c|}
\hline \multirow{3}{*}{$\begin{array}{c}\text { Analysis type of cancer } \\
\text { Bladder cancer }\end{array}$} & \multicolumn{16}{|c|}{ Cancer vs. Normal } \\
\hline & \multicolumn{2}{|c|}{$A D C Y 1$} & \multicolumn{2}{|c|}{$A D C Y 2$} & \multicolumn{2}{|c|}{$A D C Y 3$} & \multicolumn{2}{|c|}{$A D C Y 4$} & $A D C Y 5$ & \multicolumn{2}{|c|}{$A D C Y 6$} & \multicolumn{2}{|c|}{$A D C Y 7$} & $A D C Y 8$ & \multicolumn{2}{|c|}{$A D C Y 9$} \\
\hline & & & & 1 & & & & 1 & & & & & & & & 2 \\
\hline Brain and CNS cancer & & 6 & & 5 & & & 1 & & 2 & & & 1 & & 1 & & 1 \\
\hline Breast cancer & & 1 & & & & 6 & & 12 & 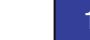 & & 1 & 3 & & & & \\
\hline Cervical cancer & & 1 & & & & & & 1 & & & & & & & & \\
\hline Colorectal cancer & & & & 3 & 6 & & & & 5 & & & & 1 & & & 11 \\
\hline Esophageal cancer & & & & & & & 2 & & & 1 & & & & & & \\
\hline Gastric cancer & & & & & 2 & & & & 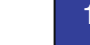 & & & & & & & \\
\hline Head and neck cancer & & & & 1 & & & & & & & & & & & & 1 \\
\hline Kidney cancer & 2 & 2 & & & 1 & & & 1 & & & & 5 & & & & \\
\hline Leukemia & 2 & & & & & & & & & & & 2 & & & 3 & \\
\hline Liver cancer & & 6 & & & & & & & & & & 1 & & & & \\
\hline Lung cancer & & 1 & & & & & & 4 & & & 1 & & 1 & & & 9 \\
\hline Lymphoma & 2 & & & & & & & & & & & & 1 & & 6 & \\
\hline Melanoma & & & 1 & & & & & & & & & & 1 & & & \\
\hline Myeloma & & & & & & & & & . & & & & & & & \\
\hline Other cancer & 1 & 1 & & 2 & & & & & & & & 2 & & & & 2 \\
\hline Ovarian cancer & & & & & & 1 & & 1 & 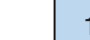 & & & & & & & 1 \\
\hline Pancreatic cancer & & 1 & & & 1 & & & & & & & 2 & & & & \\
\hline Prostate cancer & & & & & & & & & & & & & & & & \\
\hline Sarcoma & 2 & & & & & 1 & & & & & & & & & 1 & \\
\hline Significant unique analysis & 9 & 19 & 1 & 12 & 10 & 8 & 3 & 20 & 1 & 1 & 2 & 16 & 4 & 1 & 10 & 27 \\
\hline Total unique analysis & \multicolumn{2}{|c|}{428} & \multicolumn{2}{|c|}{426} & \multicolumn{2}{|c|}{372} & \multicolumn{2}{|c|}{279} & 274 & \multicolumn{2}{|c|}{418} & \multicolumn{2}{|c|}{453} & 385 & \multicolumn{2}{|c|}{411} \\
\hline
\end{tabular}

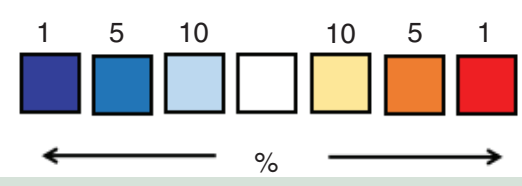

Figure 1. The mRNA levels of adenylyl cyclases in different human cancer types. The results were collected from Oncomine in cancer compared with normal tissues. The gene rank was analyzed by percentile of target gene in the top of all genes measured in each research. Red and blue color scales determined by the best gene rank percentile indicate gene expression upregulation and downregulation, respectively. The cut-off of p-value, fold change and gene ranking were defined as $0.0001,2$ and $10 \%$ respectively. 

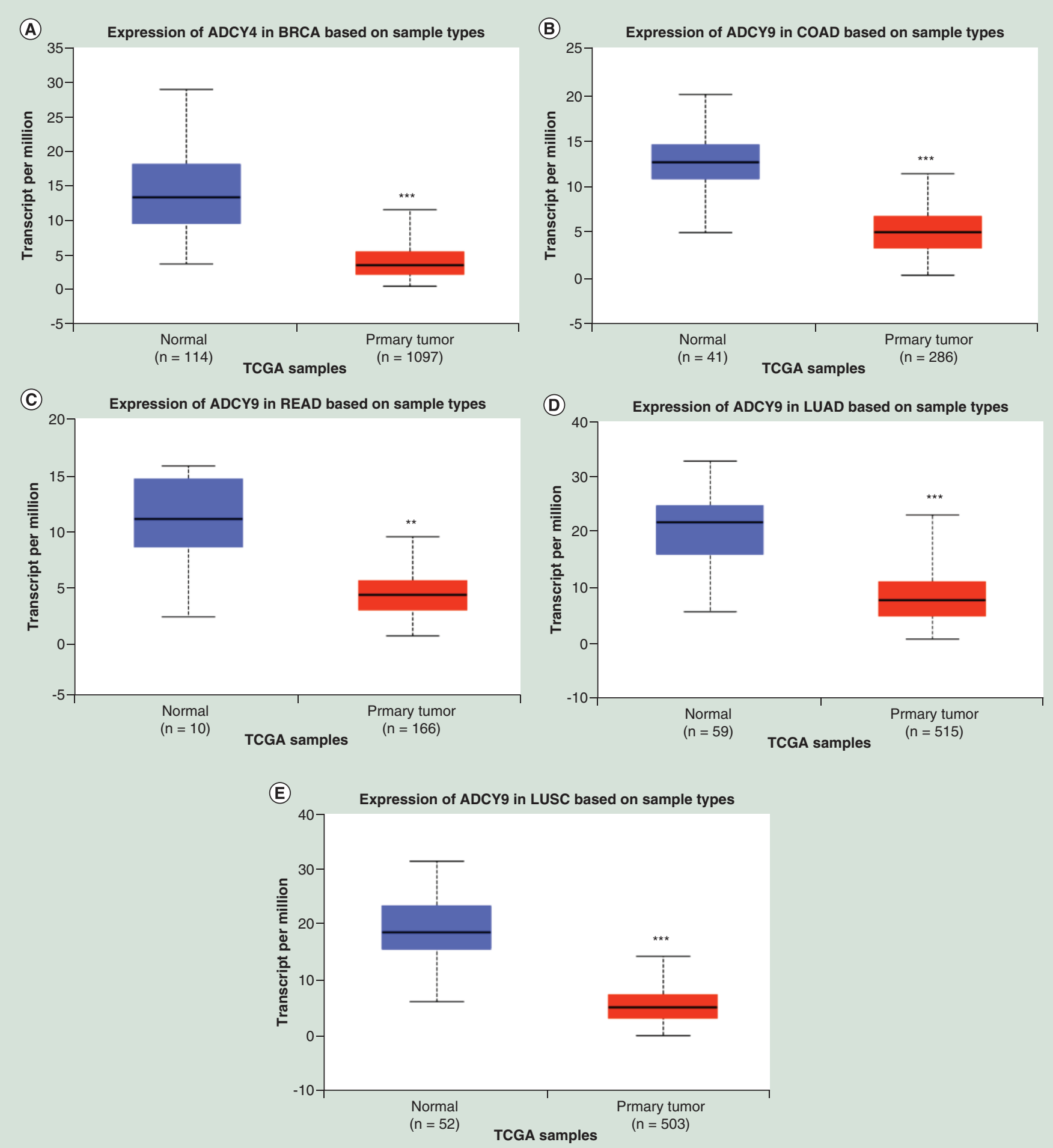

Figure 2. Box plots presenting the mRNA expression of $A D C Y 4$ and $A D C Y 9$ in breast, colorectal and lung cancer samples from UALCAN database. (A) $A D C Y 4$ in breast cancer; (B) $A D C Y 9$ in colon carcinoma; (C) $A D C Y 9$ in rectal carcinoma; (D) $A D C Y 9$ in lung adenocarcinoma; (E) $A D C Y 9$ in lung squamous cell carcinoma. $* * p<0.01 ; * * * p<0.001$. 
Table 1. The significant changes of adenylyl cyclases expression between different types of human cancer tissues in Oncomine.

\begin{tabular}{|c|c|c|c|c|c|}
\hline Gene name & Types of cancer vs normal tissues & Fold change $(>2)$ & t-test & p-value $(<1 \mathrm{E}-4)$ & Ref. \\
\hline \multirow[t]{9}{*}{$A D C Y 4$} & Invasive ductal breast carcinoma & -3.781 & -35.081 & $2.42 \mathrm{E}-82$ & [19] \\
\hline & Invasive lobular breast carcinoma & -2.747 & -18.544 & $3.01 \mathrm{E}-51$ & \\
\hline & Tubular breast carcinoma & -3.485 & -19.337 & $7.20 \mathrm{E}-40$ & \\
\hline & Mucinous breast carcinoma & -3.428 & -16.829 & $1.45 \mathrm{E}-27$ & \\
\hline & Medullary breast carcinoma & -3.937 & -16.413 & $3.55 E-21$ & \\
\hline & Invasive ductal breast carcinoma & -2.115 & -12.607 & $2.32 \mathrm{E}-21$ & TCGA \\
\hline & Invasive ductal breast carcinoma & -3.228 & -7.228 & $1.56 \mathrm{E}-7$ & [20] \\
\hline & Lobular breast carcinoma & -2.603 & -4.980 & 4.15E-5 & \\
\hline & Invasive breast carcinoma stroma & 2.271 & 5.491 & $3.82 \mathrm{E}-5$ & [18] \\
\hline \multirow[t]{17}{*}{$A D C Y 9$} & Rectal adenocarcinoma & -2.297 & -13.544 & $1.70 \mathrm{E}-26$ & [21] \\
\hline & Rectal adenocarcinoma & -3.531 & -14.235 & $8.12 \mathrm{E}-23$ & TCGA \\
\hline & Colon adenocarcinoma & -3.273 & -16.571 & 8.09E-22 & \\
\hline & Colon mucinous adenocarcinoma & -3.391 & -11.383 & $1.09 \mathrm{E}-13$ & \\
\hline & Cecum adenocarcinoma & -2.939 & -10.504 & $6.41 \mathrm{E}-13$ & \\
\hline & Rectal mucinous adenocarcinoma & -2.111 & -7.300 & $6.48 \mathrm{E}-5$ & \\
\hline & Colon adenoma & -2.161 & -13.194 & $1.19 \mathrm{E}-17$ & [22] \\
\hline & Colorectal adenocarcinoma & -2.245 & -7.266 & $2.50 \mathrm{E}-9$ & [23] \\
\hline & Colon carcinoma epithelia & -2.371 & -6.950 & $6.10 \mathrm{E}-6$ & \\
\hline & Colon carcinoma & -2.680 & -10.530 & $5.67 \mathrm{E}-8$ & [24] \\
\hline & Lung adenocarcinoma & -2.108 & -10.382 & $3.70 \mathrm{E}-17$ & [25] \\
\hline & Squamous cell lung carcinoma & -3.443 & -12.823 & $1.50 \mathrm{E}-15$ & [26] \\
\hline & Lung adenocarcinoma & -2.704 & -9.428 & $1.12 \mathrm{E}-13$ & \\
\hline & Large cell lung carcinoma & -4.230 & -9.330 & $3.73 E-9$ & \\
\hline & Small cell lung carcinoma & -8.212 & -6.256 & $3.42 \mathrm{E}-6$ & \\
\hline & Squamous cell lung carcinoma & -3.233 & -8.139 & 7.07E-5 & [28] \\
\hline & Squamous cell lung carcinoma & -2.578 & -5.379 & $9.84 \mathrm{E}-5$ & [29] \\
\hline
\end{tabular}

respectively) (Figure 2B \& 2C). Moreover, ADCY9 mRNA expression was decreased 2.89-fold and 3.40-fold in lung adenocarcinoma and squamous cell carcinoma, respectively $(\mathrm{p}=1.62 \mathrm{E}-12$ and $\mathrm{p}<1.00 \mathrm{e}-12$, respectively) (Figure 2D \& 2E).

Promoter methylation of $A D C Y 4$ negatively correlated with its mRNA expression in breast cancer DNA methylation plays an important part in epigenetic regulation of gene transcription, with promoter hypermethylation stabilizing repression of mRNA expression [9]. To assess whether ADCY4 and ADCY9 dysregulated expression was associated with DNA methylation, the MethHC database was interrogated and the promoter for $A D C Y 4$ was found to be hypermethylated in patients with breast cancer compared with normal subjects $(\mathrm{p}<0.01)$. Hypermethylation of the promoter for ADCY4 was negatively related to gene expression (Figure 3A). No such relationships were found for $A D C Y 9$ in lung cancer or colorectal cancer (Supplementary Figure 1). MEXPRESS was used to further analyze DNA methylation. In Figure 3B, the samples are presented in descending order based on the level of gene expression. ADCY4 expression in breast cancer was negatively associated (in general) with gene methylation by Pearson's correlation assessment (Figure 3B). MEXPRESS analysis found mRNA expression of ADCY4 to be lower in primary solid tumor samples than in normal tissues ( $\mathrm{p}<2.20 \mathrm{E}-12)$, and correlated with progesterone receptor $(\mathrm{p}=6.40 \mathrm{E}-05)$ and estrogen receptor status $(\mathrm{p}=1.31 \mathrm{E}-06)$. These results demonstrate $A D C Y 4$ promoter DNA hypermethylation to be associated with downregulated mRNA expression. 
(A)

Methylation and expression of ADCY4 promoter in brca

Tumor sample

Normal sample

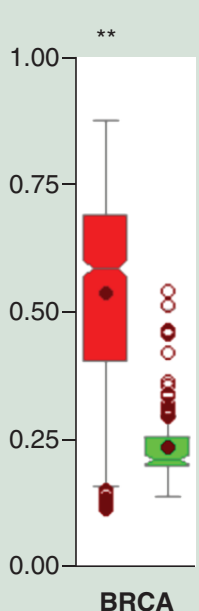

$(y=-0.000347 x+0.57399$, std err $=0.199$, corr $=0.323)$

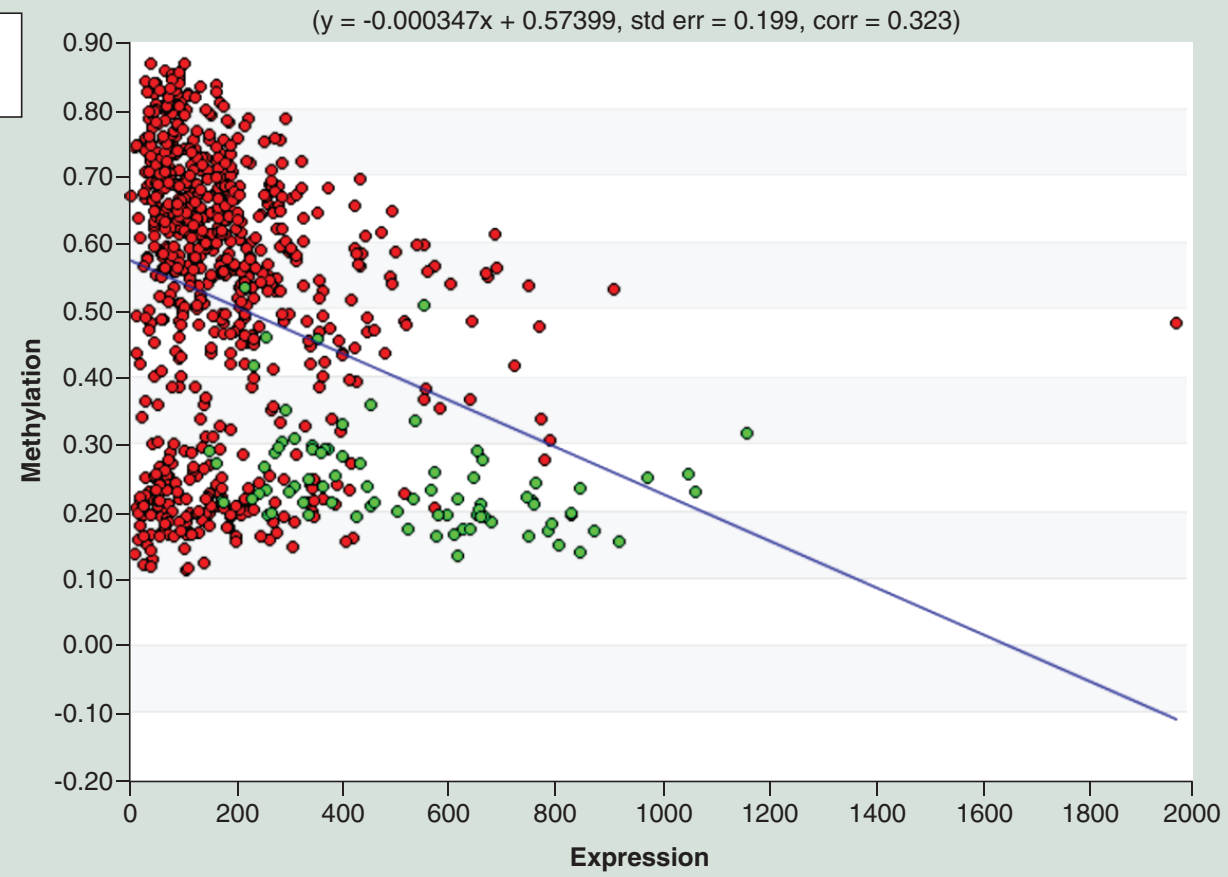

(B)

\begin{tabular}{|c|c|c|c|c|}
\hline \begin{tabular}{|l} 
Annotation \\
I Gene \\
Transcript
\end{tabular} & $\begin{array}{l}\text { Data } \\
\text { I RNA-seq v2 (log2) } \\
\text { Infinium } 450 \mathrm{k}\end{array}$ & $\begin{array}{l}\text { Statistics } \\
p \text { Wilcoxon rank-sum test } \\
p<0.05 \quad p \geq 0.05\end{array}$ & $\begin{array}{l}\text { BRCA subtype } \\
\text { | Normal } \\
\text { | Basal }\end{array}$ & $\begin{array}{l}\text { Sample type } \\
\text { | Primary solid tumor } \\
\text { | Solid tissue normal }\end{array}$ \\
\hline I $\mathrm{CPG}$ island & $\begin{array}{l}\text { Slide/clinical data } \\
\text { missing data }\end{array}$ & $\begin{array}{ccc}r \quad \text { Pearson correlation } \\
\because * & p<0.001 \\
: * & p<0.01 \\
& p<0.05 \\
& p \geq 0.05\end{array}$ & $\begin{array}{l}\text { | Her2 } \\
\text { | LumA } \\
\text { | LumB }\end{array}$ & Metastatic \\
\hline
\end{tabular}

Lymphocyte infiltration Age at diagnosis
Her2 statu

Her2 status
Pr status

Er status

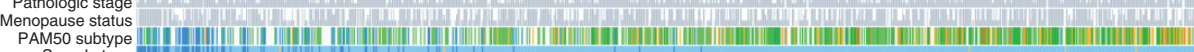

Sample type

Comparison to: expression

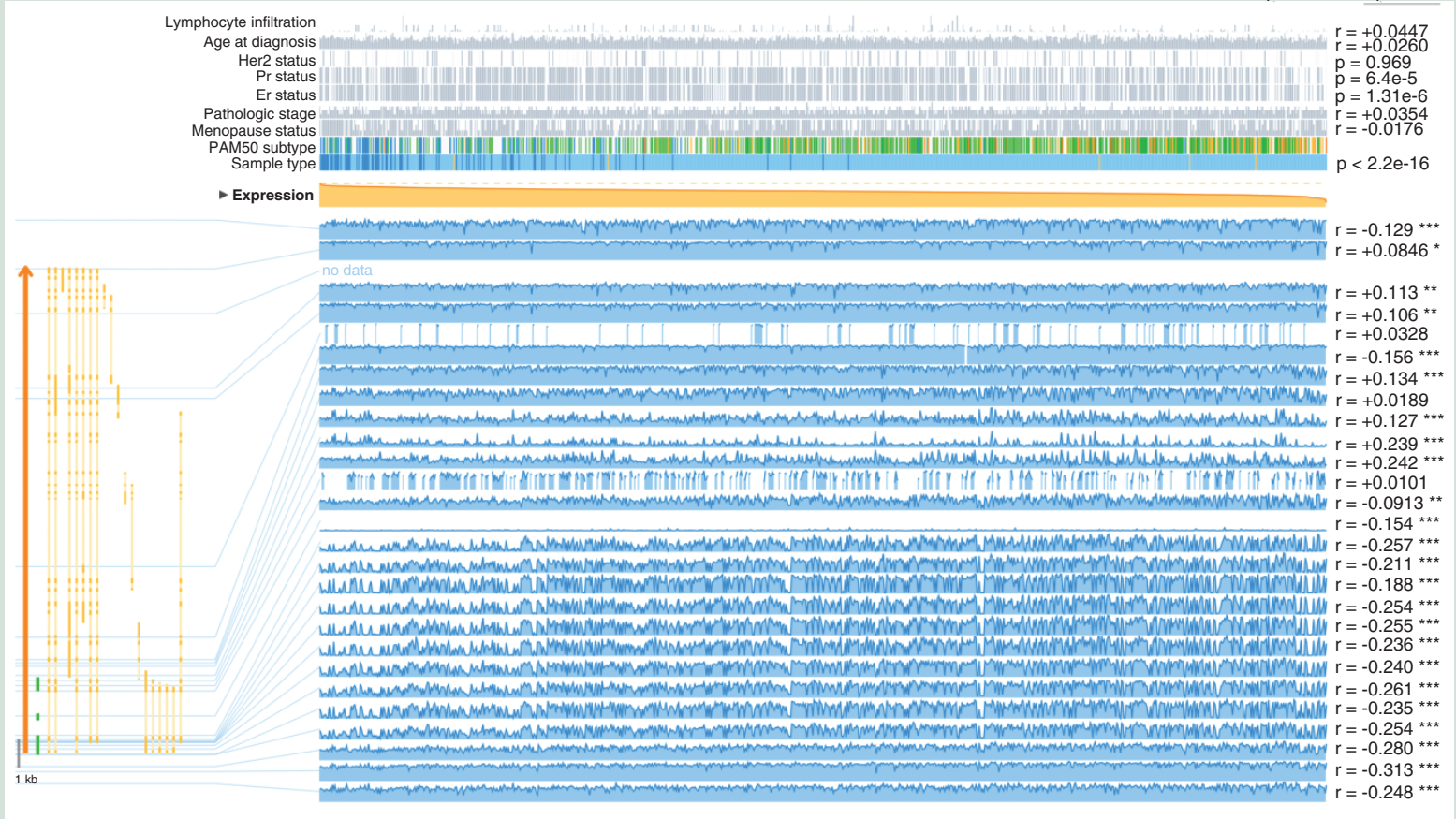

Figure 3. The epigenetic alterations on mRNA expression of $A D C Y 4$ in breast cancer. (A) The methylation levels of $A D C Y 4$ gene promoter in 735 breast invasive carcinoma and normal samples were analyzed by MethHC database. The correlation between methylation and expression of $A D C Y 4$ was also presented. ${ }^{*} \mathrm{p}<0.005$. (B) Visualization of the TCGA data for ADCY4 in breast cancer based on MEXPRESS. 871 breast invasive carcinoma samples were included. DNA methylation data for each probe were determined using Infinium HumanMethylation 450 microarray. Gene expression data were derived from IlluminaHiSeq RNASeqV2. The samples are descendingly ordered by the level of expression. The significance of the relation (correlation coefficient $r$ or $p$-value) between each row of data (clinical data, expression or methylation) was shown in the right side. ${ }^{*} p<0.05 ;{ }^{* *} p<0.01 ;{ }^{* * *} p<0.001$. 
(A)

ADCY4 1.1\% ||||||||H||||||||||||||||||||||||||||||||||||||||||||||||||||||||||||||||||||||||| Genetic alteration Missense mutation (unknown significance) | Truncating mutation (unknown significance) Amplification No alterations

(B)

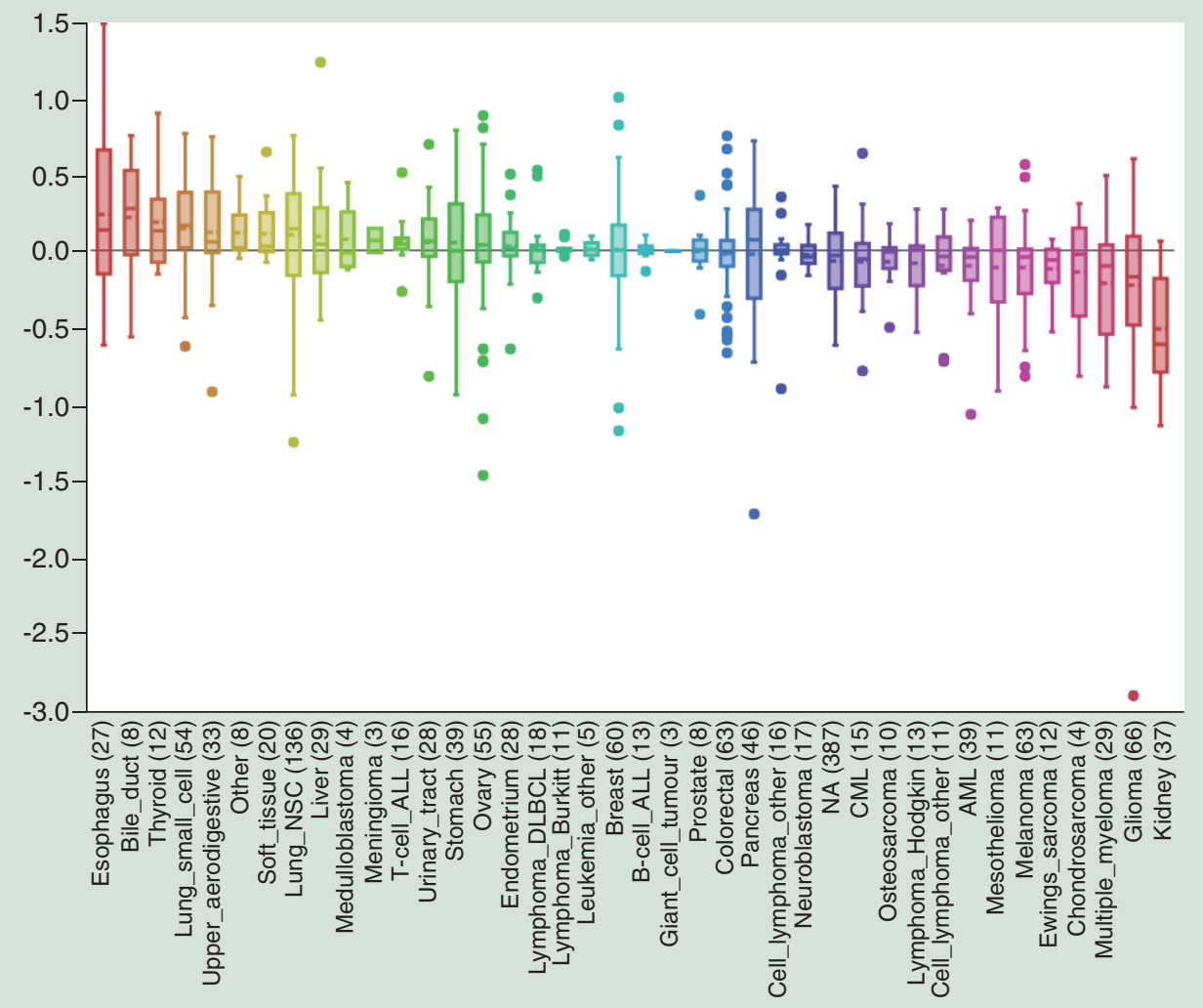

\begin{tabular}{|l|}
\hline$\square$ Esophagus (27) \\
$\square$ Bile_duct (8) \\
$\square$ Thyroid (12) \\
$\square$ Lung_small_cell (54) \\
$\square$ Upper_aerodigestive (33) \\
$\square$ Other (8) \\
$\square$ Soft_tissue (20) \\
$\square$ Lung_NSC (136) \\
$\square$ Liver (29) \\
$\square$ Medulloblastoma (4) \\
$\square$ Meningioma (3) \\
$\square$ T-cell_ALL (16) \\
$\square$ Urinary_tract (28) \\
$\square$ Stomach (39) \\
$\square$ Ovary (55) \\
$\square$ Endometrium (28) \\
$\square$ Lymphoma_DLBCL (18) \\
$\square$ Lymphoma_Burkitt (11) \\
$\square$ Leukemia_other (5) \\
$\square$ Breast (60) \\
$\square$ B-cell_ALL (13)
\end{tabular}

Figure 4. The genetic alterations on mRNA expression of $A D C Y 4$ in breast cancer. (A) OncoPrint for $A D C Y 4$ gene alterations in breast cancer. Tumor samples are shown in columns. Genomic alterations of $A D C Y 4$ are mutually exclusive. (B) The copy number levels of $A D C Y 4$ in breast cancer were relatively lower than other malignant tumors analyzed from a web portal (https://portals.broadinstitute.org/).

To examine other potential gene regulatory mechanisms, we investigated if decreased $A D C Y 4$ mRNA expression was due to genetic alterations. Gene mutations and copy-number alterations in $A D C Y 4$ were analyzed with cBioPortal. ADCY4 genetic alterations were mutually exclusive, and found in $11(1.1 \%)$ of 963 patients with invasive breast carcinoma, seven samples of which had DNA amplifications, one sample had a truncation mutation and three samples had missense mutations (Figure 4A). From a web portal, the analysis of DNA copy-number alterations demonstrated that the copy number of $A D C Y 4$ in breast cancer was relatively low compared with other malignant tumors (Figure 4B). These results demonstrate the important role for DNA methylation in the regulation of $A D C Y 4$ mRNA expression in human breast cancer.

\section{A prognostic role for $A D C Y 4$ in breast cancer patients}

Analysis of 1402 breast cancer patients by KM plotter demonstrated that high expression of $A D C Y 4$ was significantly associated with better OS (HR: 0.62 [0.45-0.85]; $\mathrm{p}=0.0029$, Figure 5A). Further, high mRNA levels of ADCY4 were associated with better RFS in 3955 breast cancer patients (HR: 0.51 [0.43-0.60]; $\mathrm{p}<1.00 \mathrm{E}-16$, Figure 5B). By use of median cut-off, $A D C Y 4$ was correlated with OS and RFS in breast cancer (HR: 0.64 [0.47-0.88]; $\mathrm{p}=$ 0.0054; HR: 0.48 [0.41-0.56]; $\mathrm{p}<1.00 \mathrm{E}-16$, respectively, Figure 5C \& 5D). Based on mRNA expression and its association with patient survival, $A D C Y 4$ may have a novel prognostic value for breast cancer patients. 
(A)

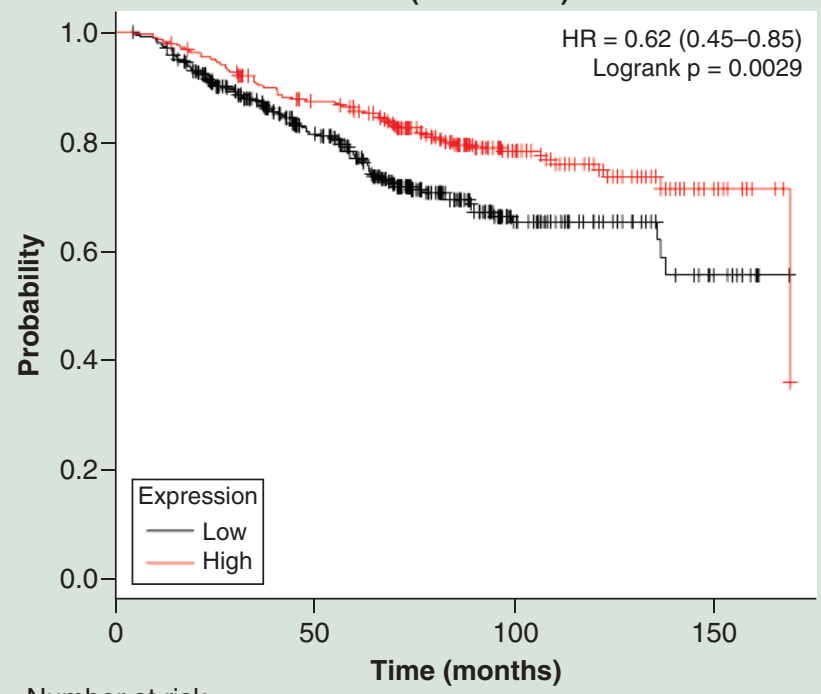

Number at risk

Low 339

High 287

(C) OS (median cutoff)

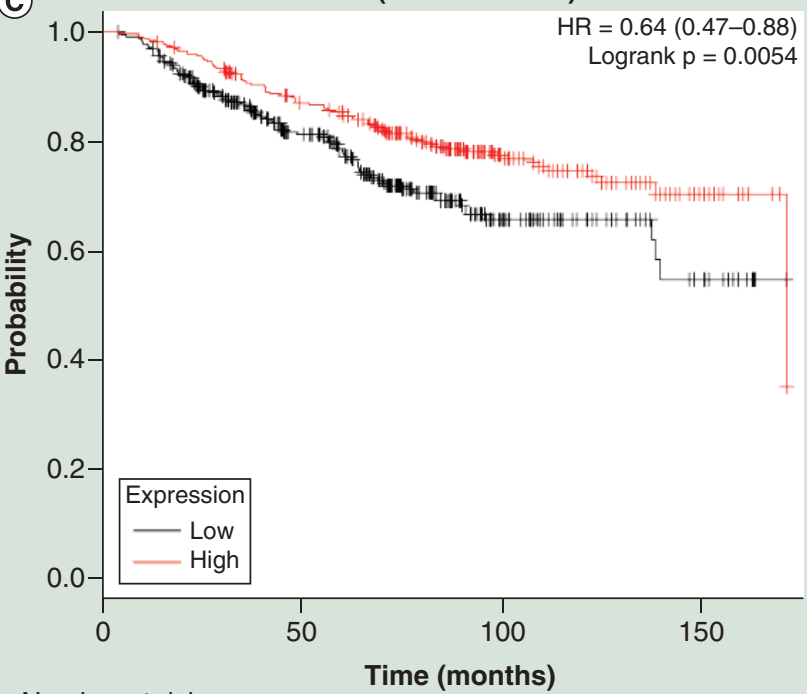

Number at risk

Low 314

High 312
214

240
10

15

(B)

RFS (best cutoff)

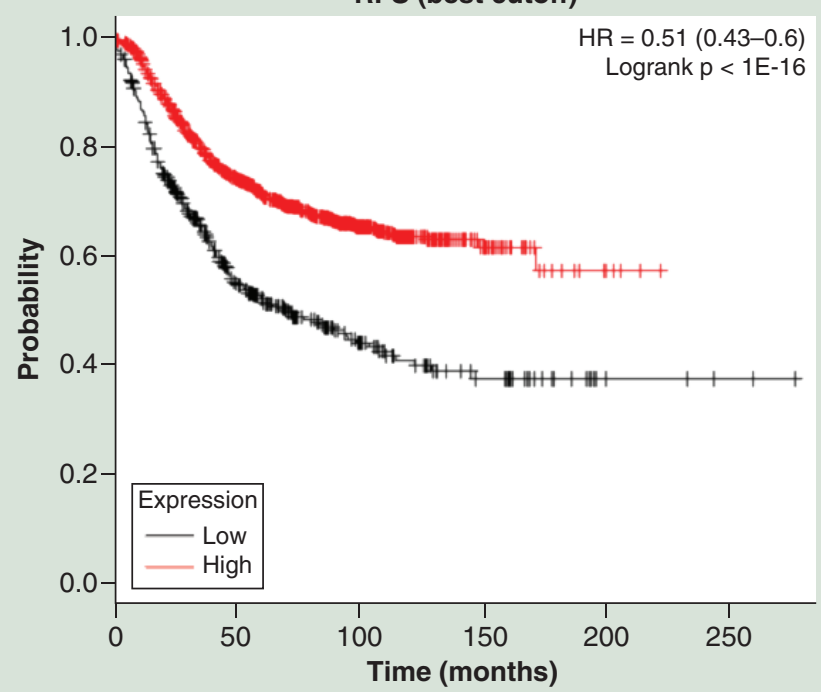

Number at risk

$\begin{array}{llclll}\text { Low } 469 & 184 & 66 & 27 & 5 & 2 \\ \text { High } 1295 & 793 & 279 & 41 & 5 & 0\end{array}$

\section{(D) RFS (median cutoff)}

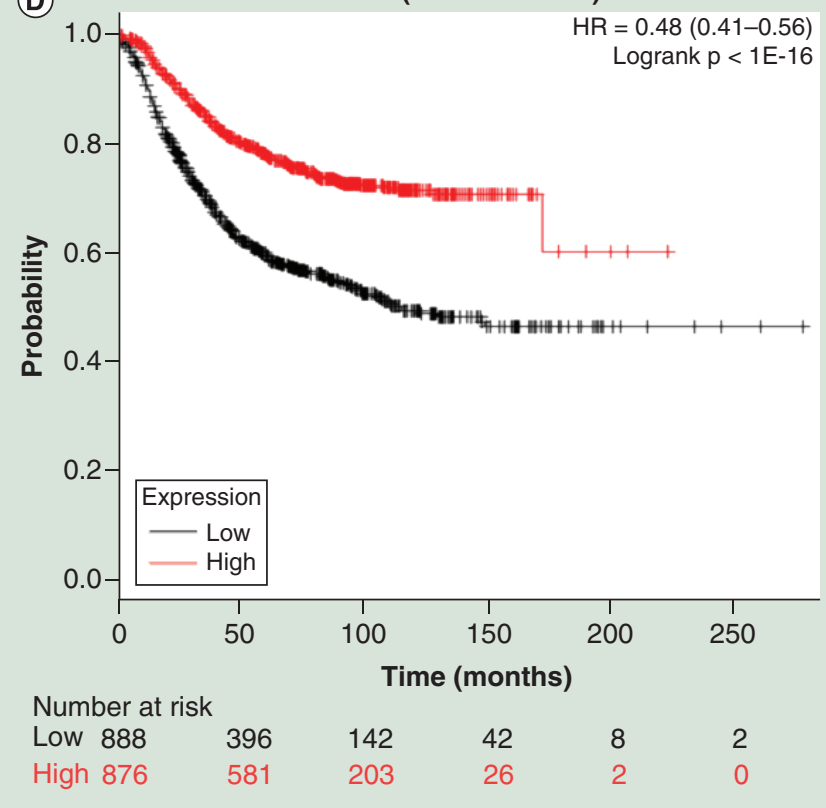

Figure 5. Prognostic values of $A D C Y 4$ for relapse-free survival and overall survival in breast cancer patients. The Kaplan-Meier plots were generated by analyzing 1105 breast invasive carcinoma patients (TCGA, Provisional, 1105 samples). p-values were calculated using log-rank test. Hazard ratios with $95 \% \mathrm{Cl}$ were presented. (A) Overall survival: splitting patients with auto select best cut-off of $A D C Y 4$; (B) relapse-free survival: splitting patients with auto select best cut-off of $A D C Y 4$; (C) OS: splitting patients with median cut-off of $A D C Y 4$; (D) relapse-free survival: splitting patients with median cut-off of $A D C Y 4$.

OS: Overall survival; RFS: Relapse-free survival.

The prognostic value of $A D C Y 4$ in breast cancer patients with different intrinsic subtypes \& tumor stages

We investigated the $A D C Y 4$ expression in TCGA samples by using UALCAN. As shown in Figure 6A, the expression of $A D C Y 4$ was significantly decreased in subclasses of luminal, HER2 positive and triple negative breast cancers 


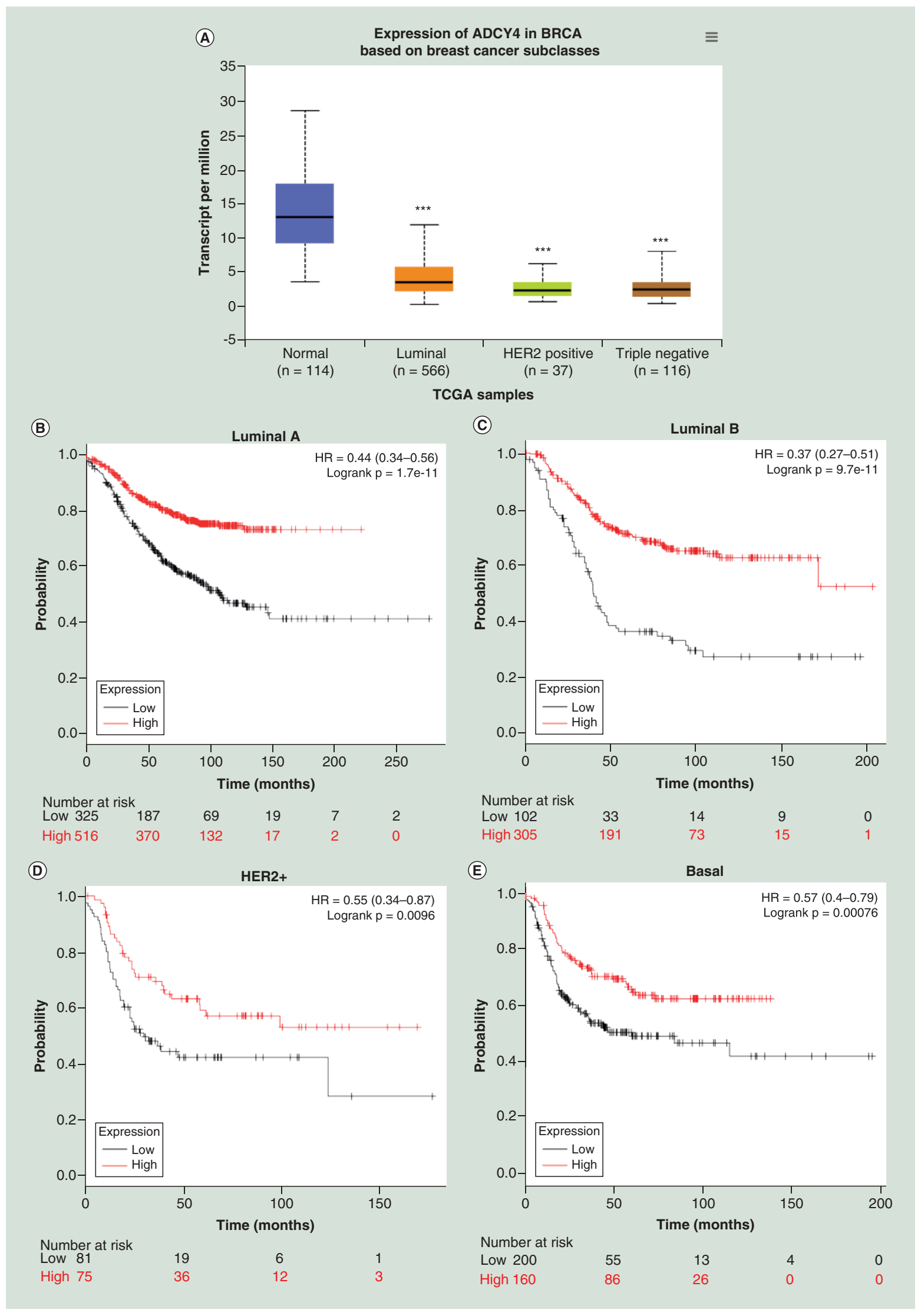

Figure 6. Prognostic values of $A D C Y 4$ in breast cancer patients with different intrinsic subtypes. (A) The mRNA levels of $A D C Y 4$ in breast cancer patients with different intrinsic subtypes, including luminal, HER2 positive and triple negative breast cancer. And the prognostic values of $A D C Y 4$ in relapse-free survival were plotted for different intrinsic subtypes of breast cancer; (B) Luminal $\mathrm{A}$ breast cancer; (C) Luminal B breast cancer; (D) HER2 ${ }^{+}$breast cancer; (E) Basal breast cancer. *** $\mathrm{p}<0.001$. 

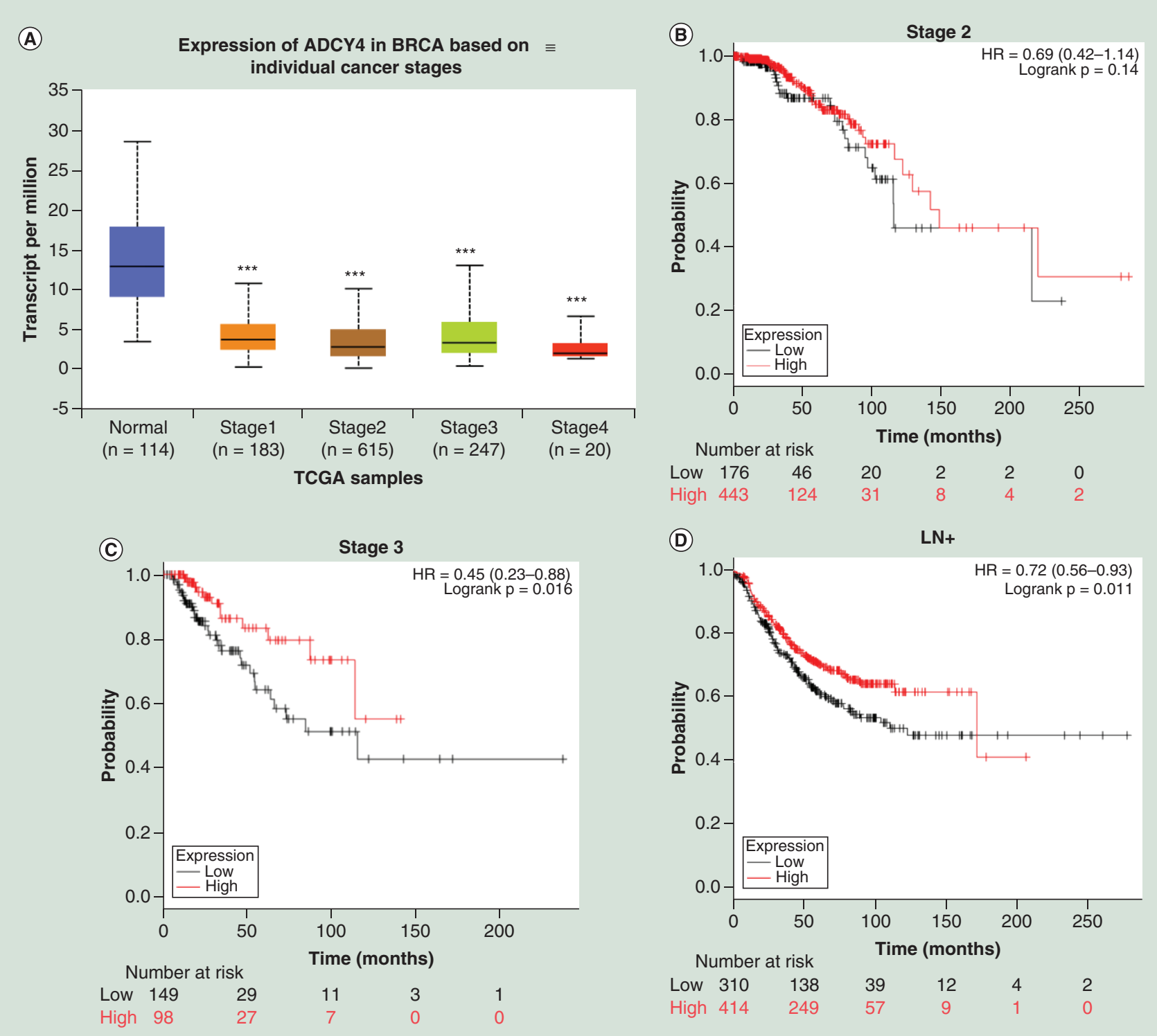

Figure 7. Prognostic values of $A D C Y 4$ in breast cancer patients with different tumor stages. (A) The mRNA expression of $A D C Y 4$ in breast cancer patients with different tumor stages. Then the prognostic values of $A D C Y 4$ were plotted for different stages of breast cancer; (B) overall survival for stage 2; (C) overall survival for stage 3; (D) relapse-free survival for lymph node positive (LN+). *** $\mathrm{p}<0.001$.

compared with normal tissue (all $\mathrm{p}<1.00 \mathrm{E}-12$ ). Moreover, the mRNA levels of $A D C Y 4$ in HER2 positive and triple negative subtypes were also lower than those in luminal $(\mathrm{p}=3.14 \mathrm{E}-08$ and $\mathrm{p}=4.42 \mathrm{E}-04$; respectively). The prognostic value for $A D C Y 4$ in different intrinsic subtypes of breast cancer was assessed by KM plotter. High expression of $A D C Y 4$ in four intrinsic subtypes was associated with better RFS prognosis (Luminal A; HR: 0.44 [0.34-0.56]; $\mathrm{p}=1.70 \mathrm{E}-11$; Luminal B; HR: $0.37(0.27-0.51) ; \mathrm{p}=9.70 \mathrm{E}-11$; HER2 ${ }^{+}$, HR: 0.55 (0.34-0.87); $\mathrm{p}=0.0096$, Basal; HR: 0.57 (0.40-0.79); $\mathrm{p}=0.00076$; respectively (Figure 6B-E).

The prognostic value of $A D C Y 4$ in breast cancer patients with different tumor stages was investigated. By analysis of the UALCAN database, we found the expression of $A D C Y 4$ to be frequently reduced in all individual cancer stages compared with normal tissue (Stage 1; $\mathrm{p}<1.00 \mathrm{E}-12$; Stage 2-4; $\mathrm{p}=1.62 \mathrm{E}-12$; respectively) (Figure 7A). Based on the KM plotter, high mRNA levels of ADCY4 were correlated with better OS in Stage 2 and 3 (Stage 2; HR: 0.69 [0.42-1.14]; $\mathrm{p}=0.14$; Stage 3; HR: 0.45 [0.23-0.88]; $\mathrm{p}=0.016$, respectively, Figure 7B \& C), and better RFS in lymph node positive patients (HR: 0.72 [0.56-0.93]; $\mathrm{p}=0.011$, Figure 7D). However, OS prognosis 
was not better for high mRNA expression of $A D C Y 4$ in patients with Stage 1 or 4 (Stage 1; HR: 5.02 [1.50-16.81]; $\mathrm{p}=0.0038$; Stage 4; HR: 2.78 [0.75-10.34]; $\mathrm{p}=0.11$, Supplementary Figure 2). Taken together, these results demonstrate a prognostic value for $A D C Y 4$ in different subgroups of breast cancer patients.

\section{Potential biomolecular networks associated with ADCY4}

To evaluate the potential role for and regulatory mechanism of ADCY4 in tumorigenesis and tumor progression, we searched ADCY4 regulated genes with STRING, with the maximum number of interactors no more than 50. We also analyzed network statistics. The number of edges was 711 , the expected number of edges was 137 , the average node degree was 27.9 , the average local clustering coefficient was 0.847 , and the protein-protein interaction enrichment value was $\mathrm{p}<1.00 \mathrm{E}-16$. Functional enrichments in this network uncovered ADCY4 to be closely associated with many genes, including ADCY9, GNAL, GCG, PRKARs, PRKACs, ADRs, ADORs, GNGs, GNAIs, DRDs and OPRs, (Figure 8A). We further performed gene annotation and pathway enrichment analysis for these genes by using Metascape. Those most significantly associated with the adenylate cyclasemodulating $G$ protein-coupled receptor signaling pathway were: ADCY9, ADORA1, ADORA2B, ADRA2A, ADRA2B, ADRA2C, ADRB1, ADRB2, AVPR2, CNR1, DRD1, DRD2, DRD3, DRD4, DRD5, GCG, GCGR, GNAI1, GNAI2, GNAI3, GNAL, GNAO1, GNAS, OPRD1, OPRK1, OPRL1, PRKACB, PTGDR, PTGER2, PTH, CALCRL and GNAT3 (Figure 8B \& Supplementary Table 1). Those most significantly associated with the cAMP signaling pathway were: ADCY9, ADORA1, ADRB1, ADRB2, DRD1, DRD2, DRD5, GNAI1, GNAI2, GNAI3, GNAS, HTR6, PRKACA, PRKACB, PRKACG, PTGER2 and VIPR2). (Figure 8B \& Supplementary Table 1). In addition, the genes of the lysophosphatidic acid receptor 4 (LPA4) pathway (ADCY9, GNAL, PRKACA) and inactivation of GLP-1 (GCG, GNB3, GNG13, GNAT3) were also significantly enriched. Each has been reported to be involved in malignant behavior and tumor progression. The results indicated that ADCY4 downregulation in breast cancer may involve the adenylate cyclase-modulating $\mathrm{G}$ protein-coupled receptor and its downstream cAMP signaling pathway.

\section{Other potential epigenetic biomarkers of ACs in common human cancers}

Lung, breast, colorectal, stomach and liver cancers are the top five cancers with the highest morbidity and mortality worldwide [1]. These were explored for prognostic biomarkers of $A C s$ downregulated by DNA methylation. First, the results of UALCAN analysis showed that downregulated mRNA levels of $A D C Y 1, A D C Y 3, A D C Y 4$ and $A D C Y 5$ were found in liver, breast, lung, and colorectal cancer tissues compared with normal tissues (liver hepatocellular carcinoma, $A D C Y 1, \mathrm{p}=0.566$; breast invasive carcinoma, $A D C Y 3, \mathrm{p}=1.62 \mathrm{E}-12$; lung adenocarcinoma and squamous cell carcinoma, $A D C Y 4, \mathrm{p}=1.53 \mathrm{E}-13$ and $\mathrm{p}<1.00 \mathrm{E}-12$, respectively; colon carcinoma and rectum carcinoma, $A D C Y 5, \mathrm{p}=1.41 \mathrm{E}-07$ and $\mathrm{p}=2.48 \mathrm{E}-03$, respectively) (Figure 9A-F). MethHC analysis demonstrated methylated $A D C Y 1, A D C Y 3, A D C Y 4$ and $A D C Y 5$ to possibly be associated with decreased mRNA expression (all $\mathrm{p}<0.005$ ) (Figure 10A-D \& Supplementary Figure 3). Finally, KM plotter analysis demonstrated high mRNA levels of $A C$ s to be significantly correlated with better OS in liver cancer patients (HR: 0.67 [0.47-0.97]; $\mathrm{p}=0.032$ ) (Figure 10E), breast cancer patients (HR: 0.64 [0.51-0.82]; $\mathrm{p}=0.00028$ ) (Figure 10F) and lung cancer patients (HR: 0.60 [0.50-0.72]; $\mathrm{p}=1.40 \mathrm{E}-08$ ) (Figure 10G). An exception was rectal carcinoma (HR: 1.84 [0.83-4.06]; $\mathrm{p}=0.13)$. Further, the prognostic value for liver, breast and lung cancer patients with different tumor stages was evaluated. As shown in Table 2, ADCY1, ADCY3 and ADCY4 demonstrated good prognostic value for some tumor stages, but with heterogeneity.

\section{Discussion}

Epigenetic modifications are increasingly identified as important targets for cancer research, including tumor initiation, which is considered regulated by both epigenetic and genetic events. In eukaryotes DNA methylation is one of the essential epigenetic mechanisms that controls cell proliferation, apoptosis, differentiation, cell cycle and transformation. Recent progress in gene promoter methylation has provided a deeper understanding of not just the mechanisms of tumorigenesis, but has also provided useful biomarkers for cancer patients [30]. Biomarkers and novel targets of human cancers are very important for diagnosis and treatment. This integrated study systematically profiled mRNA expression, genetic and epigenetic alterations, prognostic value and regulatory networks for ACs in human cancers. By analysis of the landscape of $A C$ expression in human cancer, we identified $A D C Y 4$ and $A D C Y 9$ to be frequently downregulated in several cancers, and that mRNA expression of $A D C Y 4$ and promoter hypermethylation were negatively associated in breast cancer. Moreover, high levels of $A D C Y 4$ predicted a better 
(A)

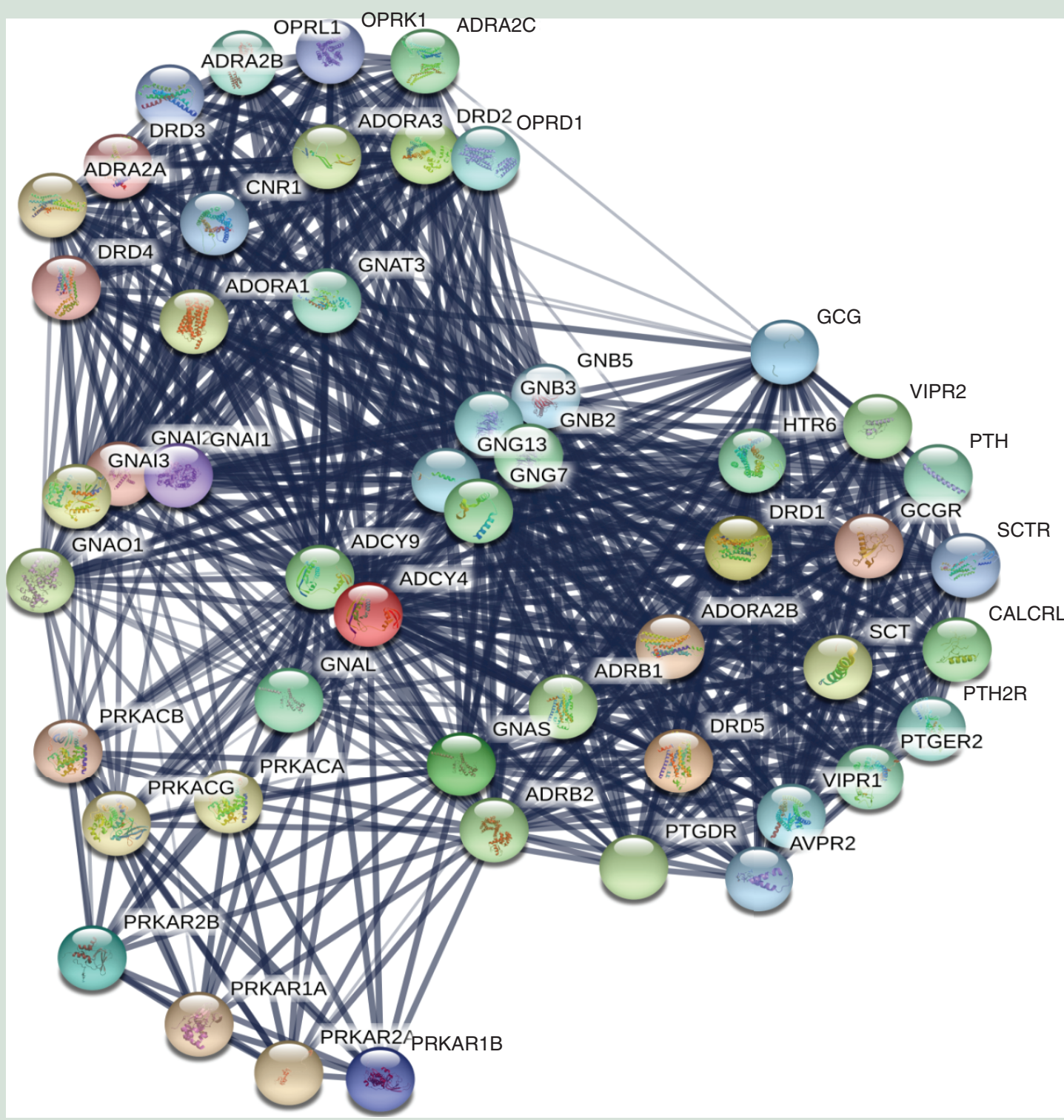

(B)

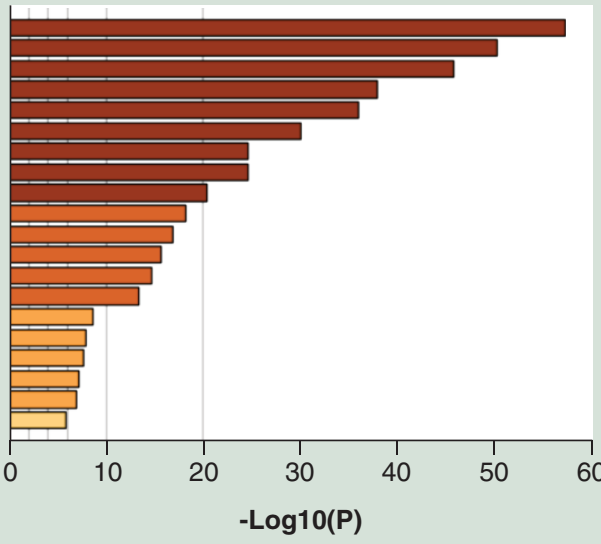

GO:0007187: G protein-coupled receptor signaling pathway, coupled to cyclic nucleotide second messenger R-HSA-500792: GPCR ligand binding

R-HSA-418594: G alpha (i) signalling events

R-HSA-418555: G alpha (s) signalling events

R-HSA-163685: integration of energy metabolism

hsa04728: dopaminergic synapse

GO:0007193: adenylate cyclase-inhibiting G protein-coupled receptor signaling pathway

R-HSA-375280:

R-HSA-375280: amine ligand-binding receptors

(1)

ho 04022.

GO:0008217:

GO:0007610: behavior

GO:0001659: temperature homeostasis

GO:0051047: positive regulation of secretion

M56: PID LPA4 pathway

R-HSA-381771: synthesis, secretion, and inactivation of glucagon-like peptide-1 (GLP-1) GO:0017158: regulation of calcium ion-dependent exocytosis GO:0042596: fear response

$-\log 10(P)$

Figure 8. ADCY4 regulated biomolecular network. (A) Protein-protein interaction networks were analyzed by Search Tool for the Retrieval of Interacting Genes/Proteins database with a confidence score of 0.4 and 50 genes interacted with ADCY 4 were identified. Line thickness indicates the strength of data support. (B) Top 20 clusters from Metascape pathway enrichment analysis of ADCY4-associated genes carried out with the following ontology sources: Kyoto Encyclopedia of Genes and Genomes Pathway, Gene Ontology Biological Processes, Reactome Gene Sets and Canonical Pathways. Length of bars represent log10 ( $p$-value) based on the best-scoring term within each cluster. 

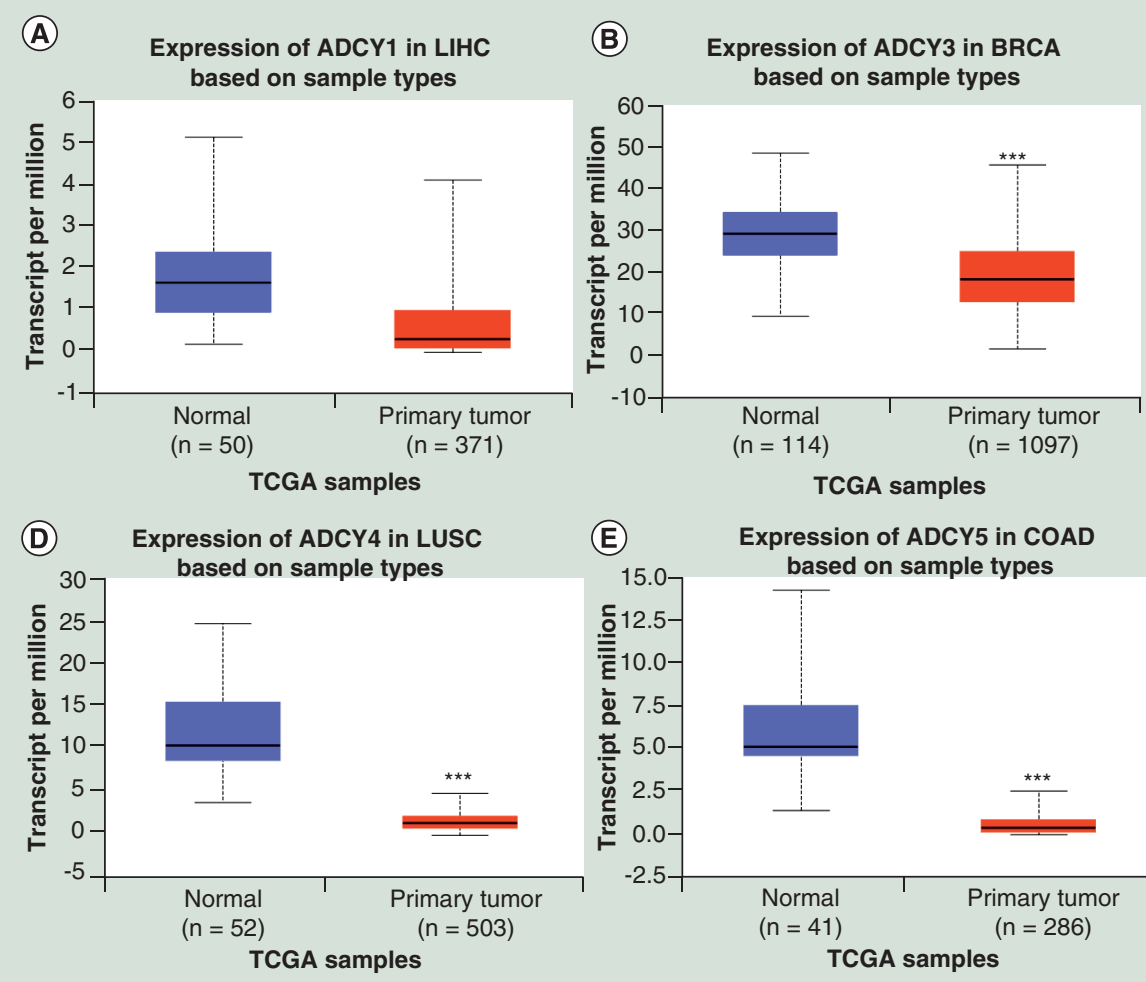
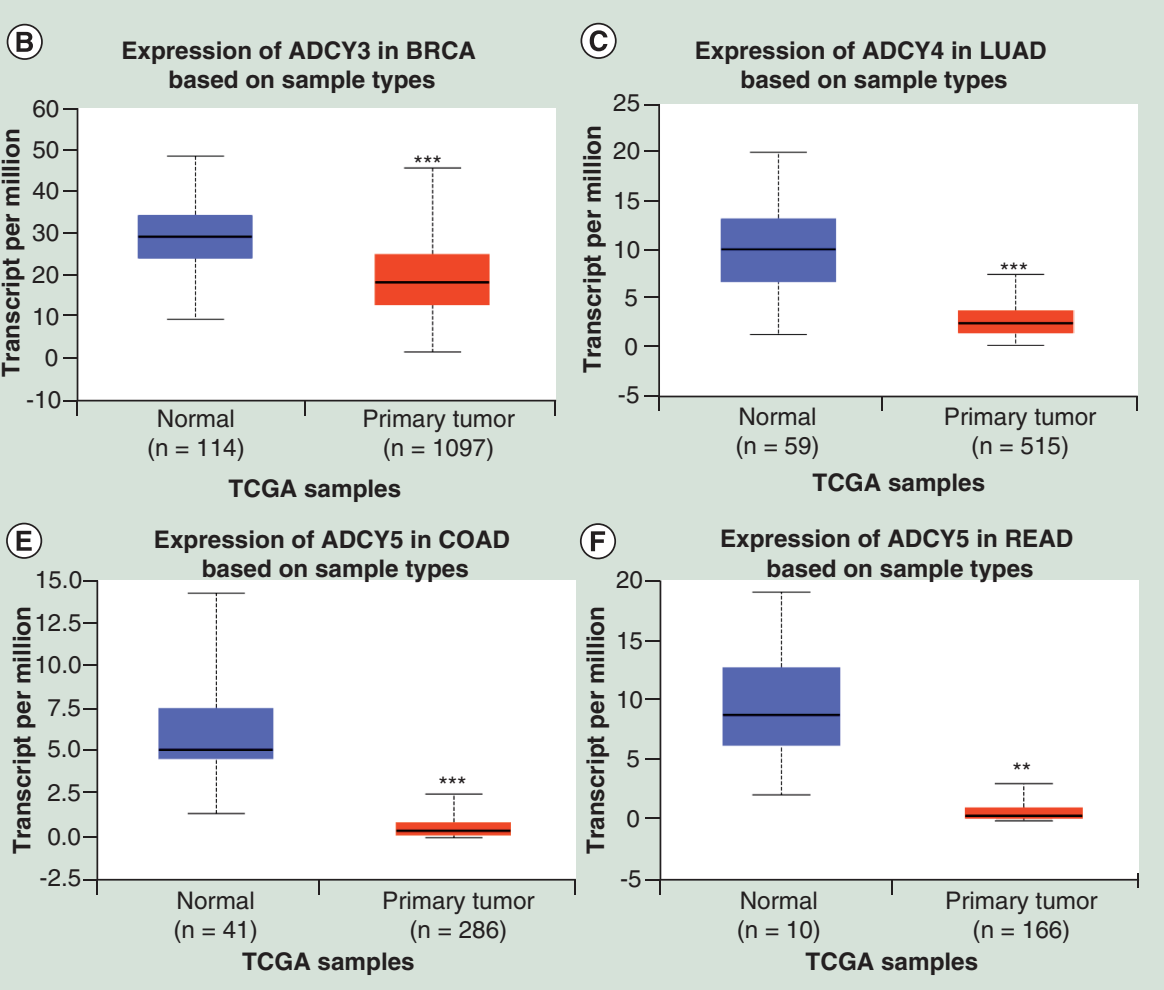

Figure 9. Box plots showing the mRNA levels of several other adenylyl cyclases in liver, breast, lung and colorectal cancer samples. (A) $A D C Y 1$ in liver hepatocellular carcinoma; (B) $A D C Y 3$ in breast invasive carcinoma; (C) $A D C Y 4$ in lung adenocarcinoma; (D) $A D C Y 4$ in lung squamous cell carcinoma; (E) $A D C Y 5$ in colon adenocarcinoma; (F) $A D C Y 5$ in rectum adenocarcinoma. ${ }^{* *} \mathrm{p}<0.01 ; * * * \mathrm{p}<0.001$.

prognosis for patients with intrinsic subtypes and tumor stages of breast cancer, with the exception of Stage 1 and 4. Consistent with $A D C Y 4$ in breast cancer, other $A C s$ ( $A D C Y 1, A D C Y 3, A D C Y 4$ and $A D C Y 5)$ were also promoter-methylated and downregulated in liver, breast, lung and colorectal cancer, respectively. High mRNA levels of these $A C s$ in liver, breast and lung cancer were significantly associated with better outcomes. However, $A C s$ were heterogeneous prognostic predictors for different stages of these cancers. Like several established biomarkers in breast cancer, cancer antigen 15-3 (CA 15-3), carcinoembryonic antigen and CA 125 [31], ADCY4 appears to be a good prognostic biomarker and to be potentially specific for human breast cancer. However, there are no studies to compare ADCY4 and common biomarkers like CA15-3 etc. Therefore, ADCY4 may be a precise diagnostic and prognostic biomarker for human breast cancer. High DNA copy number is indicative of increased levels of expression. However, several factors including gene mutation, promoter methylation, histone modifications and microRNAs may also be involved in affecting mRNA expression [32]. In this study, gene promoter DNA methylation appeared to be the key driver of decreased $A D C Y 4 \mathrm{mRNA}$ expression. Gene mutations and copy-number alterations had little effect on $A D C Y 4$ transcription.

ACs are widely expressed and play key roles in many biological processes, which precludes their use as targets for drug development, in that off target effects may result in serious side effects. However, gene knockout and transgenic animal models of most $\mathrm{AC}$ isoforms demonstrates these isoforms to have significantly different functions. ACs have been identified as potential drug targets for basic and clinical research, including neuropathy pain, neurodegenerative disease, congestive heart failure, asthma and male contraception. Corresponding compounds have been entered into clinical use [33]. At present, there are few studies of AC isoforms in human malignant tumors. Those studies focused on biomarker screening for different cancers and on discovery of interactions with other genes or small molecules. Yet, these studies did suggest the potential function and significance of ACs in human cancers [34-37].

High expression of $A D C Y 9$ has been found to be a poor prognostic factor for disease-free survival in colon cancer [38]. Human papillomavirus genotypes and DNA methylation of ADCY8, CDH8 and ZNF582 have been related to tumor cytological grade [39]. Furthermore, a lack of ADCY7 leads to inhibited growth, increased apoptosis 


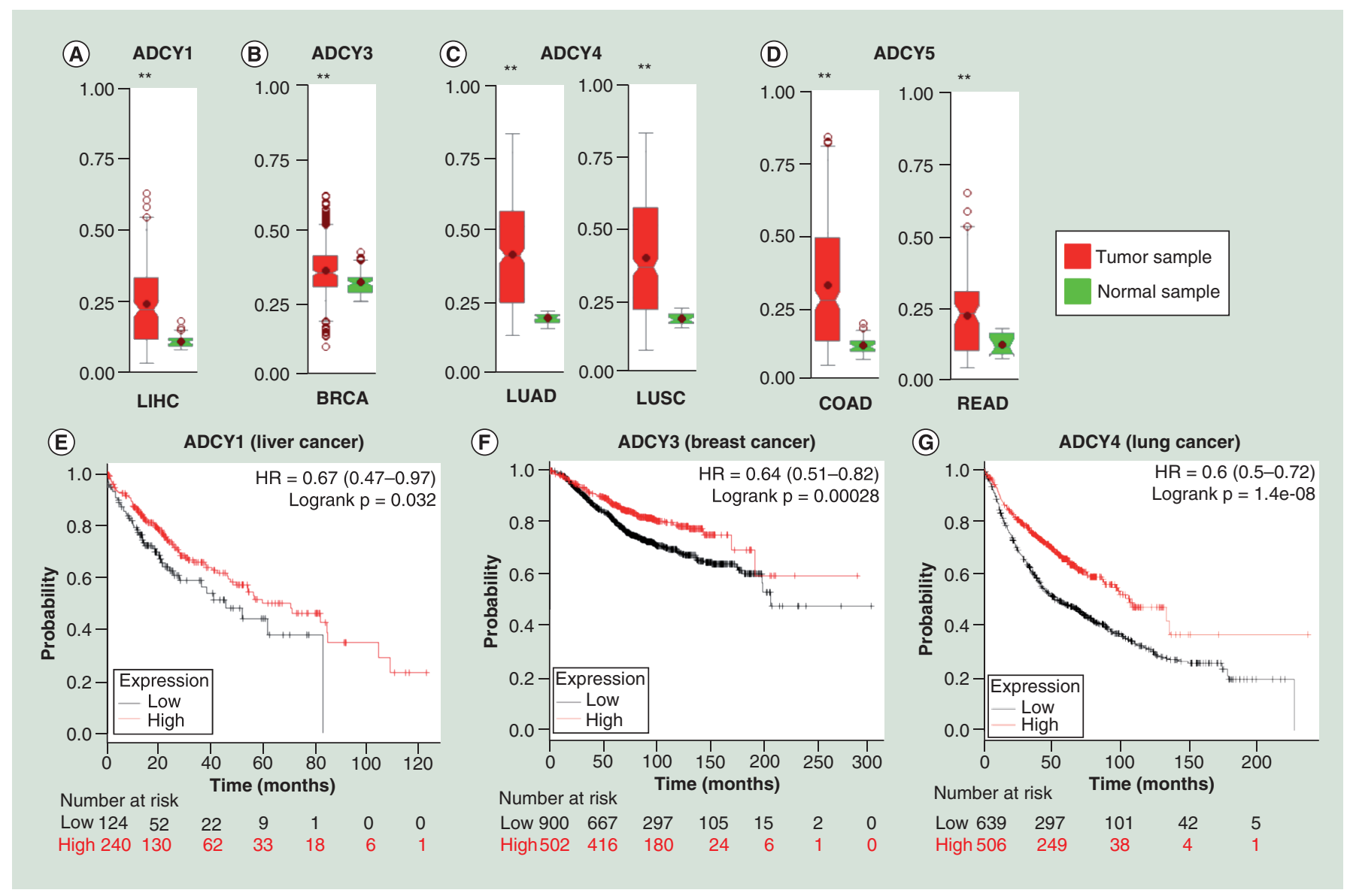

Figure 10. Prognostic values of several other adenylyl cyclases in different types of human cancers. The promoter methylation levels of adenylyl cyclases in liver hepatocellular carcinoma (A), breast invasive carcinoma (B), lung adenocarcinoma and squamous cell carcinoma (C), colon and rectum adenocarcinoma (D) and their corresponding normal samples based on MethHC database. Then we analyzed prognostic values of adenylyl cyclases in these cancers using Kaplan-Meier plotter. (E) $A D C Y 1$ in liver cancer patients. (F) $A D C Y 3$ in breast cancer patients. (G) ADCY4 in lung cancer patients. p-values were calculated by log-rank test. Hazard ratios with $95 \% \mathrm{Cl}$ are displayed. $* * p<0.005$.

and decreased expression of c-Myc, suggesting that inhibition of ADCY7 may be a new strategy for the treatment of leukemia [7]. The results of this study are consistent with another study that demonstrated ADCY3 expression to be regulated by DNA methylation. ADCY3 is an oncogene with a tumorigenic role in stomach cancer. These data suggest a new molecular target for gastric cancer, and also suggest an important role for DNA methylation in the regulation of $A C$ expression [8]. Although breast cancer studies have shown ACs to be involved in the process of tumorigenesis [40-42], there is still no direct demonstration of a molecular biological role for ACs. Analysis of ADCY4 is rare with only a single report with hepatocellular carcinoma (HCC) [43]. That report considered the clinical significance and possible molecular mechanism for the activity of endothelin receptor type B (EDNRB) and found protein interactions with KDR, VEGFC, FLT1, CDH5 and ADCY4, as the likely core EDNRB related genes. Another study identified a group of genes, including $A D C Y 4$ and $A D C Y 8$, which contain $\mathrm{CpG}$ dinucleotides that discriminated superclusters of highly and moderately methylated breast cancer samples and a possible association with metabolic enzymes and their regulators [44]. This study has also shown that ADCY4 plays a potential role in energy metabolism (Figure 8B; R-HSA-163685). Further, ADCY4 expression was repressed by promoter hypermethylation in breast cancer.

By analysis of related genes, previously unidentified biological signaling pathways can be identified in human cancers. Functional enrichment analysis of ADCY4-related genes identified pathways that may play key roles in tumorigenesis and progression. As expected, genes associated with $G$ protein-coupled receptors and the downstream cAMP signaling pathway were found to be enriched. Importantly, newly identified LPA4 and GLP-1 may provide 


\begin{tabular}{|c|c|c|c|c|c|c|c|}
\hline $\begin{array}{l}\text { Cancer type/gene } \\
\text { symbol }\end{array}$ & $\begin{array}{l}\text { Patient number at } \\
\text { risk }\end{array}$ & $\begin{array}{l}\text { AC expression } \\
\text { level }\end{array}$ & Stage & HR & $95 \% \mathrm{Cl}$ & $\begin{array}{l}\text { Prognostic } \\
\text { outcome }\end{array}$ & p-value \\
\hline \multirow{4}{*}{$\begin{array}{l}\text { Liver } \\
\text { cancer/ADCY1 }\end{array}$} & 110 & Low & 1 & 1.97 & $1.07-3.63$ & Poor & 0.027 \\
\hline & 60 & High & & & & & \\
\hline & 47 & Low & 3 & 0.33 & $0.17-0.64$ & Good & 0.00063 \\
\hline & 36 & High & & & & & \\
\hline \multirow{5}{*}{$\begin{array}{l}\text { Breast } \\
\text { cancer } / A D C Y 3\end{array}$} & 113 & Low & 1 & 0.36 & $0.1-1.26$ & Good & 0.094 \\
\hline & 67 & High & & & & & \\
\hline & 143 & High & & & & & \\
\hline & 14 & Low & 4 & 2.59 & $0.75-8.94$ & Poor & 0.12 \\
\hline & 6 & High & & & & & \\
\hline \multirow{6}{*}{$\begin{array}{l}\text { Lung } \\
\text { cancer/ADCY4 }\end{array}$} & 135 & Low & 1 & 0.31 & $0.23-0.42$ & Good & $5 E-15$ \\
\hline & 314 & High & & & & & \\
\hline & 113 & Low & 2 & 0.83 & $0.49-1.41$ & Good & 0.49 \\
\hline & 48 & High & & & & & \\
\hline & 24 & Low & 3 & 0.71 & $0.35-1.45$ & Good & 0.34 \\
\hline & 20 & High & & & & & \\
\hline
\end{tabular}

insight into the discovery of associated molecular mechanisms and may encourage further consideration in human cancers.

Upstream signaling is achieved by changing the activity of ACs, which is dependent upon interaction with another protein, usually the coupling of a $\mathrm{G}$ protein subunit to a membrane receptor [4]. Interestingly, the $\mathrm{Gi}$ $\alpha$ protein is highly expressed in breast cancer cells, particularly in metastatic breast cancer cells [45]. The Gi $\alpha$ protein is an important regulator of RTK signal transduction in normal and breast cancer cells, with regulatory effects on Akt, mTORC1 and Erk1/2 activation when combined with growth factors [45]. The results of this study suggest a potential relationship between ADCY4 and the Gi protein (inhibitory G protein) $\alpha$ subunit (Figure 8B; R-HSA-418594). Another study has generated new approaches for drug discovery exploiting the auto-regulatory molecular mechanism of the ADCY9-Gs (stimulated G protein) $\alpha$ structure [46]. We also found a relationship between ADCY4 and the Gs $\alpha$ protein (Figure 8B; R-HSA-418555). Furthermore, the proximal targets of cAMP are proteins with various functions, although somewhat similar in sequence. These cAMP-binding proteins control the activity of PKA, guanine nucleotide exchange factor and ion channels, regulating the transcription rate of many genes, including transcription factors that play biological roles in mesenchymal-to-epithelial transition, inhibition of cell growth, migration and sensitivity to antitumor drugs [3-5].

Tumor blood vessels impact the tumor microenvironment, with vascular normalization an important means by which to improve drug delivery and anti-tumor immunity [47,48]. However, angiogenesis inhibitors often induce hypoxia, which may promote tumor progression [48]. Signals mediated by LPA4 facilitate network formation of tumor vessels that restore normal vascular function permitting drug delivery [49]. Based on protein-protein interaction identified by the STRING database and Metascape analysis, ADCY4 may be associated with the LPA4 pathway (Figure 8B; M56). LPA4 has been shown to promote the formation of fine vascular structures within brain tumors. With regard to mechanism, RhoA/ROCK activation contributes to LPA-induced endothelial adhesion, regulates VCAM-1 expression and induces increased lymphocyte infiltration around the tumor. LPA4 can improve the delivery of exogenous IgG into tumors and enhance the effect of anti-PD-1 therapy [49,50]. Moreover, LPAs are 
extracellular biological lipids that interact with $\mathrm{G}$ protein coupled receptors [51] and can activate G-protein-coupled receptors of the endothelial differentiation gene (Edg) family (LPA1, LPA2 and LPA3) and non-Edg gene family (LPA4, LPA5 and LPA6) [52]. LPA4, LPA5 and LPA6 have been shown to be involved in tumor progression of pancreatic cancer cells [53]. In head and neck squamous cell carcinoma (HNSCC), LPA signaling mediated by both Edg and non-Edg receptors may potentially modulate malignant behavior [54].

Another association with ADCY4 was synthesis, secretion and inactivation of GLP-1 (Figure 8B; R-HSA381771). GLP-1 is a type of gastrointestinal hormone that enhances glucose-dependent insulin secretion and suppresses glucagon release [55]. Breast cancer is one of the most common cancers in female patients who have Type 2 diabetes mellitus and obesity [56]. A previous report has demonstrated an anti-cancer effects for GLP-1 receptor (GLP-1R) agonist exendin-4 [57]. In breast cancer, GLP-1 has been shown to attenuate cell proliferation through activation of GLP-1R and inhibition of NF- $\mathrm{B}$ [58]. An anticancer effect for selected diabetic patients with ovarian cancer was found for GLP-1R agonists [59]. In prostate cancer, GLP-1 analogs that bind GLP-1R attenuate growth by regulation of the P38 pathway [60].

There are limitations to this study. First, this analysis is based on mRNA levels derived from public datasets and confirmation at the protein levels is necessary. Besides, some bioinformatics tools based on TCGA and GEO datasets and used in our study only have limiting function like hierarchical analysis, without multivariable Cox regression analysis. Second, although our study emphasizes the prognostic value of ADCY4 in breast cancer and predicts several potential signaling pathways, further experiments are needed to clarify specific underlying mechanisms by which ADCY4 affects cell growth, apoptosis and metastasis. Further, ADCY4 is a membrane-integral AC [6]. Hence, the specific structure and function of ADCY4-G protein-coupled receptors may provide a theoretical basis for new approaches to drug discovery. It is therefore important to determine how ADCY4 impacts drug delivery and anti-tumor immunity in select diabetic patients with human cancers.

\section{Conclusion}

In summary, for the first time this study comprehensively and systematically investigated the potential role and functional networks of ACs in human cancers based on public datasets. Our work uncovered a novel impact of epigenetic and genetic alterations on gene mRNA expression. These findings suggest that ADCY4 may function as a candidate tumor suppressor gene. Hence, its biologic role in tumorigenesis and cancer progression is worthy of further study. Based on the data herein, ADCY4 may be used as a prognostic biomarker and therapeutic target for human breast cancer.

\section{Future perspective}

$\mathrm{AC}$ isoforms are potential targets in many human diseases, and some compounds have been entered the clinical use. Our work for the first time reveals the epigenetic regulation mechanisms and potential biologic roles of ACs in human cancers, especially ADCY4 in breast cancer. Therefore, it is urgent to do basic experiments and clinical trials to dig deeply their molecular mechanisms and application values. We are planning to collect breast cancer samples to confirm and evaluate the potential values of ADCY4 as a biomarker in the next step. Although selective AC isoform target drugs are still in the early stages of development, the selective drugs have significant advantages in side effects, and ACs may eventually be considered as a viable therapeutic and epigenetic target in human cancers.

\footnotetext{
Author contributions

Y Fan and Q Wen conceived and designed the study. J Mu, M Huang, S Lin and J Fan obtained the data. J Mu, M Huang, S Imani, and $Y$ Wang performed data analysis. $Y$ Fan and $S$ Imani drafted the manuscript. All authors reviewed the manuscript and approved the final version before submission.
}

Financial \& competing interests disclosure

The present work was supported by the Research Foundation of Southwest Medical University for Youth (grant no. 090300030685), the Funded Project of Affiliated Hospital of Southwest Medical University for Doctors (grant no. 17135) and the National Natural Science Foundation of China (grant no. 81201682). The authors have no other relevant affiliations or financial involvement with any organization or entity with a financial interest in or financial conflict with the subject matter or materials discussed in the manuscript apart from those disclosed.

No writing assistance was utilized in the production of this manuscript. 


\section{Open access}

This work is licensed under the Attribution-NonCommercial-NoDerivatives 4.0 Unported License. To view a copy of this license, visit http://creativecommons.org/licenses/by-nc-nd/4.0/

\section{Summary points}

- We evaluated the mRNA expression and DNA methylation profiles of adenylyl cyclases in human cancers using public databases and found the $A D C Y 4$ and $A D C Y 9$ expression was frequently downregulated in several tumor types.

- Genetic and epigenetic analysis uncovered promoter hypermethylation of $A D C Y 4$ downregulated its mRNA levels in breast cancer.

- The high level of $A D C Y 4$ mRNA expression was identified to significantly relate with better survival in breast cancer, and could have prognostic values in different subgroup patients with different tumor stages and intrinsic subtypes.

- We proved that ADCY4 notably associated with adenylate cyclase-modulating G protein-coupled receptor and its downstream CAMP signaling pathway and possibly affected cell growth, apoptosis and metastasis through LPA4 and GLP-1 mechanisms.

- Other adenylyl cyclase isoforms were also confirmed to have epigenetic and prognostic values in lung, breast and liver cancers.

\section{References}

Papers of special note have been highlighted as: $\bullet$ of interest; $\bullet \bullet$ of considerable interest

1. Bray F, Ferlay J, Soerjomataram I, Siegel RL, Torre LA, Jemal A. Global cancer statistics 2018: GLOBOCAN estimates of incidence and mortality worldwide for 36 cancers in 185 countries. CA Cancer J. Clin. 68(6), 394-424 (2018).

2. Hanahan D, Weinberg RA. Hallmarks of cancer: the next generation. Cell 144(5), 646-674 (2011).

3. Dong H, Claffey KP, Brocke S, Epstein PM. Inhibition of breast cancer cell migration by activation of cAMP signaling. Breast Cancer Res. Treat. 152(1), 17-28 (2015).

4. Gancedo JM. Biological roles of cAMP: variations on a theme in the different kingdoms of life. Biol. Rev. Cambridge Philosoph. Soc. 88(3), 645-668 (2013).

5. Pattabiraman DR, Bierie B, Kober KI et al. Activation of PKA leads to mesenchymal-to-epithelial transition and loss of tumor-initiating ability. Science 351(6277), aad3680 (2016).

6. Pierre S, Eschenhagen T, Geisslinger G, Scholich K. Capturing adenylyl cyclases as potential drug targets. Nat. Rev. Drug Discov. 8(4), 321-335 (2009).

7. Li C, Xie J, Lu Z et al. ADCY7 supports development of acute myeloid leukemia. Biochem. Biophys. Res. Commun. 465(1), 47-52 (2015).

8. Hong SH, Goh SH, Lee SJ et al. Upregulation of adenylate cyclase 3 (ADCY3) increases the tumorigenic potential of cells by activating the CREB pathway. Oncotarget 4(10), 1791-1803 (2013).

9. Xiang TX, Yuan Y, Li LL et al. Aberrant promoter CpG methylation and its translational applications in breast cancer. Chin. J. Cancer 32(1), 12-20 (2013).

-• DNA methylation and its roles in breast cancer.

10. Rhodes DR, Yu J, Shanker K et al. ONCOMINE: a cancer microarray database and integrated data-mining platform. Neoplasia 6(1), $1-6$ (2004).

11. Chandrashekar DS, Bashel B, Balasubramanya SaH et al. UALCAN: A Portal for Facilitating Tumor Subgroup Gene Expression and Survival Analyses. Neoplasia 19(8), 649-658 (2017).

12. Huang WY, Hsu SD, Huang HY et al. MethHC: a database of DNA methylation and gene expression in human cancer. Nucleic Acids Res. 43(Database Issue), D856-D861 (2015).

13. Koch A, De Meyer T, Jeschke J, Van Criekinge W. MEXPRESS: visualizing expression, DNA methylation and clinical TCGA data. BMC Genomics 16, 636 (2015).

14. Cerami E, Gao J, Dogrusoz U et al. The cBio cancer genomics portal: an open platform for exploring multidimensional cancer genomics data. Cancer Discov. 2(5), 401-404 (2012).

15. Gyorffy B, Lanczky A, Eklund AC et al. An online survival analysis tool to rapidly assess the effect of 22,277 genes on breast cancer prognosis using microarray data of 1,809 patients. Breast Cancer Res. Treat. 123(3), 725-731 (2010).

16. Szklarczyk D, Franceschini A, Wyder S et al. STRING v10: protein-protein interaction networks, integrated over the tree of life. Nucleic Acids Res. 43(Database Issue), D447-D452 (2015). 
17. Zhou Y, Zhou B, Pache L et al. Metascape provides a biologist-oriented resource for the analysis of systems-level datasets. Nat. Commun. 10(1), 1523 (2019).

\section{- Analysis tools used in this study}

18. Finak G, Bertos N, Pepin F et al. Stromal gene expression predicts clinical outcome in breast cancer. Nat. Med. 14(5), 518-527 (2008).

19. Curtis C, Shah SP, Chin SF et al. The genomic and transcriptomic architecture of 2,000 breast tumours reveals novel subgroups. Nature 486(7403), 346-352 (2012).

20. Zhao H, Langerod A, Ji Y et al. Different gene expression patterns in invasive lobular and ductal carcinomas of the breast. Mol. Biol. Cell 15(6), 2523-2536 (2004).

21. Gaedcke J, Grade M, Jung K et al. Mutated KRAS results in overexpression of DUSP4, a MAP-kinase phosphatase, and SMYD3, a histone methyltransferase, in rectal carcinomas. Genes Chromosomes Cancer 49(11), 1024-1034 (2010).

22. Sabates-Bellver J, Van Der Flier LG, De Palo M et al. Transcriptome profile of human colorectal adenomas. Mol. Cancer Res. MCR 5(12), 1263-1275 (2007).

23. Skrzypczak M, Goryca K, Rubel T et al. Modeling oncogenic signaling in colon tumors by multidirectional analyses of microarray data directed for maximization of analytical reliability. PLoS ONE 5(10), (2010).

24. Zou TT, Selaru FM, Xu Y et al. Application of cDNA microarrays to generate a molecular taxonomy capable of distinguishing between colon cancer and normal colon. Oncogene 21(31), 4855-4862 (2002).

25. Landi MT, Dracheva $\mathrm{T}$, Rotunno $\mathrm{M}$ et al. Gene expression signature of cigarette smoking and its role in lung adenocarcinoma development and survival. PLoS ONE 3(2), e1651 (2008).

26. Hou J, Aerts J, Den Hamer B et al. Gene expression-based classification of non-small cell lung carcinomas and survival prediction. PLoS ONE 5(4), e10312 (2010).

27. Bhattacharjee A, Richards WG, Staunton J et al. Classification of human lung carcinomas by mRNA expression profiling reveals distinct adenocarcinoma subclasses. Proc. Natl Acad. Sci. USA 98(24), 13790-13795 (2001).

28. Wachi S, Yoneda K, Wu R. Interactome-transcriptome analysis reveals the high centrality of genes differentially expressed in lung cancer tissues. Bioinformatics 21(23), 4205-4208 (2005).

29. Garber ME, Troyanskaya OG, Schluens K et al. Diversity of gene expression in adenocarcinoma of the lung. Proc. Natl Acad. Sci. USA 98(24), 13784-13789 (2001).

- The expression of adenylyl cyclases (ACs) in human cancer based on microarray data.

30. Pan Y, Liu G, Zhou F, Su B, Li Y. DNA methylation profiles in cancer diagnosis and therapeutics. Clin. Exp. Med. 18(1), 1-14 (2018).

31. Li L, Gao Q, Xu G et al. Postoperative recurrence analysis of breast cancer patients based on clinical serum markers using discriminant methods. Cancer Biomark. 19(4), 403-409 (2017).

32. Sharma S, Kelly TK, Jones PA. Epigenetics in cancer. Carcinogenesis 31(1), 27-36 (2010).

33. Dessauer CW, Watts VJ, Ostrom RS, Conti M, Dove S, Seifert R. International union of basic and clinical pharmacology. CI. Structures and small molecule modulators of mammalian adenylyl cyclases. Pharmacol. Rev. 69(2), 93-139 (2017).

-. ACs as drug targets in human diseases.

34. Zhang Y, Fang L, Zang Y, Xu Z. Identification of core genes and key pathways via integrated analysis of gene expression and DNA Methylation profiles in bladder cancer. Med. Sci. Monit. 24, 3024-3033 (2018).

35. Wen DY, Lin P, Liang HW et al. Up-regulation of CTD-2547G23.4 in hepatocellular carcinoma tissues and its prospective molecular regulatory mechanism: a novel qRT-PCR and bioinformatics analysis study. Cancer Cell Int. 18, 74 (2018).

36. Choi M, Kadara H, Zhang J et al. Mutation profiles in early-stage lung squamous cell carcinoma with clinical follow-up and correlation with markers of immune function. Ann. Oncol. 28(1), 83-89 (2017).

37. Tang J, Kong D, Cui Q et al. Bioinformatic analysis and identification of potential prognostic microRNAs and mRNAs in thyroid cancer. PeerJ 6, e4674 (2018).

38. Yi H, Wang K, Jin JF et al. Elevated adenylyl cyclase 9 expression is a potential prognostic biomarker for patients with colon cancer. Med. Sci. Monit. 24, 19-25 (2018).

39. Shen-Gunther J, Wang CM, Poage GM et al. Molecular pap smear: HPV genotype and DNA methylation of ADCY8, CDH8, and ZNF582 as an integrated biomarker for high-grade cervical cytology. Clin. Epigenet. 8, 96 (2016).

40. Del Valle PR, Milani C, Brentani MM et al. Transcriptional profile of fibroblasts obtained from the primary site, lymph node and bone marrow of breast cancer patients. Genet. Mol. Biol. 37(3), 480-489 (2014).

41. Li Y, Liu X, Tang H, Yang H, Meng X. RNA sequencing uncovers molecular mechanisms underlying pathological complete response to chemotherapy in patients with operable breast cancer. Med. Sci. Monit. 23, 4321-4327 (2017).

42. Schwickert A, Weghake E, Bruggemann $\mathrm{K}$ et al. microRNA miR-142-3p Inhibits breast cancer cell invasiveness by synchronous targeting of WASL, integrin alpha V, and additional cytoskeletal elements. PLoS ONE 10(12), e0143993 (2015). 
43. Zhang L, Luo B, Dang YW et al. The clinical significance of endothelin receptor type B in hepatocellular carcinoma and its potential molecular mechanism. Exp. Mol. Pathol. 107, 141-157 (2019).

44. Tanas AS, Sigin VO, Kalinkin AI et al. Genome-wide methylotyping resolves breast cancer epigenetic heterogeneity and suggests novel therapeutic perspectives. Epigenomics 11(6), 605-617 (2019).

- $\quad$ ADCY4 is possibly associated with metabolism.

45. Wang Z, Dela Cruz R, Ji F et al. G(i)alpha proteins exhibit functional differences in the activation of ERK1/2, Akt and mTORC1 by growth factors in normal and breast cancer cells. Cell Commun. Signal. CCS 12, 10 (2014).

-. Gi proteins and its function in breast cancer.

46. Qi C, Sorrentino S, Medalia O, Korkhov VM. The structure of a membrane adenylyl cyclase bound to an activated stimulatory G protein. Science 364(6438), 389-394 (2019).

- The association of Gs protein and AC isoform.

47. Jain RK. Antiangiogenesis strategies revisited: from starving tumors to alleviating hypoxia. Cancer cell 26(5), 605-622 (2014).

48. Jain RK. Normalization of tumor vasculature: an emerging concept in antiangiogenic therapy. Science 307(5706), 58-62 (2005).

49. Takara K, Eino D, Ando K et al. Lysophosphatidic acid receptor 4 activation augments drug delivery in tumors by tightening endothelial cell-cell contact. Cell Rep. 20(9), 2072-2086 (2017).

50. Eino D, Tsukada Y, Naito $\mathrm{H}$ et al. LPA4-mediated vascular network formation increases the efficacy of anti-PD-1 therapy against brain tumors. Cancer Res. 78(23), 6607-6620 (2018).

51. Yung YC, Stoddard NC, Chun J. LPA receptor signaling: pharmacology, physiology, and pathophysiology. J. Lipid Res. 55(7), 1192-1214 (2014).

52. Choi JW, Herr DR, Noguchi K et al. LPA receptors: subtypes and biological actions. Annu. Rev. Pharmacol. Toxicol. 50, 157-186 (2010).

53. Ishii S, Hirane M, Fukushima K, Tomimatsu A, Fukushima N, Tsujiuchi T. Diverse effects of LPA4, LPA5 and LPA6 on the activation of tumor progression in pancreatic cancer cells. Biochem. Biophys. Res. Commun. 461(1), 59-64 (2015).

54. Matayoshi S, Chiba S, Lin Y et al. Lysophosphatidic acid receptor 4 signaling potentially modulates malignant behavior in human head and neck squamous cell carcinoma cells. Int. J. Oncol. 42(5), 1560-1568 (2013).

- The roles of LPA4 and its signaling in cancer.

55. Ravassa S, Zudaire A, Diez J. GLP-1 and cardioprotection: from bench to bedside. Cardiovasc. Res. 94(2), 316-323 (2012).

56. Noto H, Goto A, Tsujimoto T, Osame K, Noda M. Latest insights into the risk of cancer in diabetes. J. Diabetes Investig. 4(3), 225-232 (2013).

57. Nomiyama T, Kawanami T, Irie S et al. Exendin-4, a GLP-1 receptor agonist, attenuates prostate cancer growth. Diabetes 63(11), 3891-3905 (2014).

58. Iwaya C, Nomiyama T, Komatsu $S$ et al. Exendin-4, a glucagonlike peptide-1 receptor agonist, attenuates breast cancer growth by inhibiting NF-kappaB activation. Endocrinology 158(12), 4218-4232 (2017).

59. He W, Yu S, Wang L et al. Exendin-4 inhibits growth and augments apoptosis of ovarian cancer cells. Mol. Cell. Endocrinol. 436, 240-249 (2016).

60. Li XN, Bu HM, Ma XH et al. Glucagon-like peptide-1 analogues inhibit proliferation and increase apoptosis of human prostate cancer cells in vitro. Exp. Clin. Endocrinol. Diabetes 125(2), 91-97 (2017).

- GLP-1: from diabetes to cancer. 